\title{
Strategic Performance of Deferred Acceptance in Dynamic Matching Problems ${ }^{\dagger}$
}

\author{
By John Kennes, Daniel Monte, and Norovsambuu Tumennasan*
}

In dynamic matching problems, priorities often depend on previous allocations and create opportunities for manipulations that are absent in static problems. In the dynamic school choice problem, students can manipulate the period-by-period deferred acceptance (DA) mechanism. With a commonly used restriction on the schools' priorities, manipulation vanishes as the number of agents increases, but without it the mechanism can be manipulated, even in large economies. We also check manipulation in large finite economies through a novel computer algorithm, which can check every possible manipulation by examining all the different matchings that a single player can induce. (JEL C78, I21, I28)

$\checkmark$ his paper proposes a market design solution to dynamic matching problems. Many important assignment markets are inherently dynamic because some agents repeatedly participate in the assignment process. A few notable examples are the following: the problem of allocating children to public day care centers, where the same child is assigned to day care centers in consequent periods; the centralized assignment of teachers to public schools, and on-campus housing assignments. ${ }^{1,2}$ In practice, even the school choice problem has dynamic features: Families with multiple children participate in the assignment of schools several times. ${ }^{3}$

An important feature present in many dynamic markets is that the priorities of one side of the market is history-dependent. For example, in both the day

\footnotetext{
* Kennes: Department of Economics and Business, Aarhus University, Fuglesangs Allé 4, DK-8210 Aarhus V, Denmark (email: jkennes@econ.au.dk); Monte: Sao Paulo School of Economics-FGV, Rua Itapeva, 474-12 andar, Bela Vista, CEP 01332-000, Sao Paulo, Brazil (email: daniel.monte@fgv.br); Tumennasan: Department of Economics, Dalhousie University, 6406 University Ave, Halifax, Canada (email: norov@ dal.ca). Michael Ostrovsky was coeditor for this article. We thank the financial support from the Center for Research in the Foundations of Electronic Markets. Tumennasan thanks the Economics Departments at Harvard University and Boston College for its hospitality and the Danish Council for Independent Research for providing the travel grant (\#12-128681). André Diniz and Jan Kjærgaard provided excellent research assistance. We thank Eduardo Azevedo, Francis Bloch, Thomas Gall, Fuhito Kojima, Maciej Kotowski, Jacob Leshno, Sangram Kadam, and Juan Pereyra as well as several seminar audiences.

Go to https://doi.org/10.1257/mic.20170077 to visit the article page for additional materials and author disclosure statement(s) or to comment in the online discussion forum.

${ }^{1}$ See Kennes, Monte, and Tumennasan (2014) for detailed information on the current Danish daycare system, Pereyra (2013) for dynamic teacher assignment, and Kurino (2014) for dynamic housing assignment.

${ }^{2}$ Indeed, there is a myriad of examples of dynamic matching. In addition to those listed above, one can think of the allocation process in the Army, the allocation of public sector workers among different offices, and the allocation of diplomats in different embassies.

${ }^{3}$ See Dur (2011) for school choice with siblings.
} 
care assignment and the teacher assignment problems a child or teacher cannot be involuntarily displaced from a school to which she is currently assigned. This history dependence creates opportunities for manipulations that are absent in static problems. An agent might misreport her preferences to affect the priority ranking and to receive a better allocation in the future. This opportunity for manipulations implies that results in dynamic-market design are mostly negative. Kennes, Monte, and Tumennasan (2014) proved an impossibility result: There does not exist any mechanism that is both stable and strategy-proof. This leaves open the important market design question of how to operate such markets.

Our model is a school choice problem in which children live for two periods and schools are infinitely lived. We assume throughout the paper that preferences are rankable, in the sense that they are separable over time and consistent across periods. ${ }^{4}$ We consider two commonly used classes of priorities of schools over children in the paper, and show that they capture two strikingly different aspects of the problem. First, we consider a class of strict priorities that are history-dependent only through Guaranteed Continued Enrollment - that is, a student who has been allocated to a school will have the highest priority in that school. We say that this class of priorities satisfies independence of previous assignment since priorities do not change at schools in which the agent was not assigned to. Second, we consider a class of priorities in which, additionally to the guaranteed continued enrollment, there is a second channel in which past allocations affect current priorities. Namely, an agent can have a higher priority at some schools if she stays home in the first period. This is motivated by the assignment system of young children to public day care centers in Denmark, which is one of the practical dynamic matching problems we have in mind. In the Danish system, it is often the case that a day care institution gives high priority to a child who was eligible to participate in the assignment system in the previous period, but nevertheless was not allocated to any day carethis child receives a higher priority than those who attended any day care institution other than the one in question. This rule is denoted "child care guarantee." Thus, for lack of a better label, we denote this priority system as Danish priorities.

Our paper studies the strategic performance of the period-by-period deferred acceptance (DA) mechanism in dynamic markets under these two priority systems. The DA has played a prominent role in the school choice problem-the static problem most closely related to ours. Our results show that the way priorities are dynamically updated according to previous matchings are of paramount importance for the strategic performance of the DA in large economies. We provide two sets of results under the two priority systems (i) we prove that under independence of previous assignment the DA is approximately strategy-proof in large markets, whereas it remains manipulable under Danish priorities, and (ii) we provide computer simulations to show that finite markets with similar size to markets in common applications are very rarely manipulable under independence of previous assignment, but remain manipulable under Danish priorities.

\footnotetext{
${ }^{4}$ This does not exclude the possibility of switching costs or status quo biases. Rankability is similar to responsiveness (see Roth and Sotomayor 1990). Without rankability, it can be shown that a stable matching might not exist in dynamic markets.
} 
The positive results of the strategic performance of DA under independence of previous assignment justify the use of this mechanism and priority system in our environment. There is a wide range of markets that might benefit from this positive result. The most natural application is the assignment of young children to day care centers, and the day care system is public and centralized in most European countries and many other places. Another natural application of our results is on the problem of assigning teachers to public schools.

To better understand the intuition behind these two contrasting results, obtained under the two different priority systems, let us illustrate their main features using the two examples below. In both examples we assume that children live for two periods and that schools have unit capacity.

Example 1 (Priorities: Guaranteed Continued Enrollment): David is born in period 0, Ana and Bob are born in period 1, while Chris is born in period 2. There are three schools, $s_{1}, s_{2}$, and $s_{3}$. David's favorite school is $s_{1}$ and $s_{1}$ gives David the highest priority among all four children. Thus, David will be allocated to $s_{1}$ in period 1. Let us assume that preferences over the different pairs of schools (one school per period) for Ana, Bob, and Chris are (partially) represented by the following table:

$\begin{array}{ll}\text { Ana: } & s_{1} \succ s_{2} \succ s_{3} \\ \text { Bob: } & s_{3} \succ s_{1} \succ s_{2} . \\ \text { Chris: } & s_{3} \succ s_{1} \succ s_{2}\end{array}$

The information in the table is a partial representation of the preferences of the students. For example, Ana's preferences are such that she would prefer to be allocated in $s_{1}$ for both periods than to be allocated in $s_{2}$ for both periods, which in turn is better than being placed in $s_{3}$ for both periods. Let us also assume that she would prefer to be allocated to $s_{3}$ and $s_{1}$ instead of $s_{2}$ for both periods (this information is not in the table). Bob and Chris have identical preferences and they rank $s_{3}$ as the best school, followed by $s_{1}$, and only then by $s_{2}$. This is the only information we will need for the purpose of this example. The schools' priorities for incoming students are such that Ana is the preferred student at schools $s_{2}$ and $s_{3}$, while Chris is the preferred student at school $s_{1}$ and is ranked higher than Bob at schools $s_{2}$ and $s_{3}$. We represent the priorities below:

\begin{tabular}{|c|c|c|c|}
\hline Chris & $\triangleright$ & Ana & $\triangleright$ \\
\hline Ana & $\triangleright$ & Chris & $D$ \\
\hline Ana & $\triangleright$ & Chris & $\triangleright$ \\
\hline
\end{tabular}

We also assume that priorities in a given period depend on the previous allocation only through Guaranteed Continued Enrollment; a student placed in any given school will have the highest priority in that school in the subsequent period. In a static DA mechanism, Ana and Bob would report the following preferences in the 
first period $s_{1} \succ s_{2} \succ s_{3}$ and $s_{3} \succ s_{1} \succ s_{2}$, respectively. Using DA, we can see that Ana will be placed at $s_{2}$, while Bob is placed at $s_{3}$. In the following period, Ana and Bob repeat their reports and Chris reports $s_{3} \succ s_{1} \succ s_{2}$. Given that Bob was allocated at $s_{3}$, Guaranteed Continued Enrollment implies that he will have the highest priority in that school, and will remain there. Ana will be placed at $s_{2}$ and Chris at $s_{1}$. This describes the final outcome under truth-telling of this simple economy. Suppose that Ana, instead of reporting $s_{1} \succ s_{2} \succ s_{3}$ in the first period, reports $s_{1} \succ s_{3} \succ s_{2}$. She will be placed at $s_{3}$ and Bob will go to $s_{2}$. In the second period, Ana reports her truthful preferences $s_{1} \succ s_{2} \succ s_{3}$. Ana's final allocation is $\left(s_{3}, s_{1}\right)$ to which she prefers when compared to her allocation under truth-telling: $\left(s_{2}, s_{2}\right)$.

In the example above, adapted from Kennes, Monte, and Tumennasan (2014), Ana gained from not telling the truth about what we denote, for now, as her static preferences. That is, in period 1 she reported $s_{3}$ as a better school than $s_{2}$, which is not true. This triggered a sequence of events. Bob was not able to get to his best school and instead went to $s_{2}$. Because of this, in period 2, Bob and Chris competed for school $s_{3}$ and Chris had a higher priority. This leaves school $s_{1}$ free for Ana to come. Note that when Ana reports her true static preference, Bob is allocated to $s_{3}$ and due to Guaranteed Continued Enrollment, he has a higher priority than Chris in period 2, so Chris has to go to his second best school, $s_{1}$, forcing Ana back to school $s_{2}$. The mechanism is manipulable, in the sense that reporting their true static preference is not a weakly dominant strategy. 5

Let us now look at a second example, which incorporates Danish priorities.

Example 2 (Danish Priorities): Camilla is born in period 0, Anna is born in period 1, and Bo is born in period 2. There are two schools: $s_{1}$ and $s_{2}$. Camilla has the highest priority in school $s_{1}$, her favorite one. Let $h$ represent the option of staying home. Anna's preference profile can be partially represented by

Anna: $\left(s_{1}, s_{1}\right) \succ\left(s_{2}, s_{1}\right) \succ\left(h, s_{1}\right) \succ\left(s_{2}, s_{2}\right) \succ\left(h, s_{2}\right) \succ(h, h)$.

Bo prefers $s_{1}$ over $s_{2}$. The priorities assigned to the schools are such that Bo has a higher priority than Anna in any school. However, priorities might change depending on previous allocation. They might change due to guaranteed continued enrollment, or if a student stayed home in the previous period. Specifically, every student will have the highest priority in the school they were allocated in the previous period, and they will have the highest priority among newcomers in any given school if they were eligible for school in the previous period but were not allocated to any school in the system. With this priority system, if Anna reports truthfully, she will be allocated to $\left(s_{2}, s_{2}\right)$. In the second period she competes with Bo for a spot at $s_{1}$, but

\footnotetext{
${ }^{5}$ Indeed this incentive for manipulation is present for another celebrated mechanism, the Top-Trading Cycles. In fact, a stronger result is proved in Monte and Tumennasan (2015), the only mechanism that is Pareto efficient, non-bossy, and strategy-proof is the sequential dictatorship.
} 
Bo has a higher priority. If, instead, Anna reports home as being preferred to $s_{2}$ she will be allocated to $h$ in the first period and will have a higher priority than Bo in the second period, so her final allocation will be $\left(h, s_{1}\right)$, which she prefers over $\left(s_{2}, s_{2}\right)$.

The example above captures the fact that manipulating under Danish priorities does not have to lead to a chain of events, and in this sense it is easier than manipulating the mechanism under independence of previous assignment. In contrast to Example 1, here Anna simply stays home and this gives her a higher priority in her favorite school in period 2.

In our large market session, the growth of the market we consider here is the same as the one in Azevedo and Leshno (2016): the set of agents along with the capacities of the schools (not the set of schools) increases. Under this growth dynamic we identify the conditions for the implementation of the DA mechanism as the number of participants increases. We show that if each schools' priorities over agents depend on the previous history only through previously enrolled agents- the condition that also guarantees the stability of the DA mechanism (Kennes, Monte, and Tumennasan 2014) - then the DA mechanism is approximately strategy-proof in large markets. Specifically, in Theorem 4, we show that the fraction of agents who may have incentives to misreport their preferences when all other participants are reporting truthfully approaches zero as the market size tends to infinity.

On the other hand, if the deferred acceptance mechanism is applied period-by-period in a market with Danish priorities, the system remains manipulable even in large markets. One intuition for these strikingly different results is the following. The DA is known to be strategy-proof in static problems, thus, given that preferences are rankable, a successful manipulation must imply an improvement in the second-year allocation (at the cost of a weakly worse first-year allocation). In order to improve her second-year allocation, the agent must manipulate her report in the first year in such a way that priorities for the second period change to benefit her. If the priority system is such that the only history-dependence is through guaranteed continued enrollment, the agent cannot change her own priority at a school other than the one she attends in her first period. Thus, a successful manipulation must mean that she was able to change other agents' priorities at schools. As the market gets large, changing her report does not help her to affect the threshold priorities of the schools. On the other hand, Danish priorities implies that an agent can change her priority at different schools simply by a unilateral move, that is, by staying home. This move does not depend on the size of the market. Thus, these contrasting results highlight the fact that our large market result is not simply a consequence of the fact that the agent is too small, but rather it is dependent on the specific priority system in place.

We then proceed through simulations to show how often agents have an incentive to manipulate the DA mechanism under the two priority systems that we study. There is, however, a significant computational complexity. To illustrate, consider a market with 30 schools. In this market, any given agent has a strategy set of 30 ! elements (more than $10^{32}$ elements). Thus, checking each possible manipulation is not a feasible task. In static marriage problems it is well-known that to evaluate whether an agent, say a woman, has an incentive to manipulate the men-proposing DA mech- 
anism it suffices to check the truncation strategies-those in which the woman only reduces her acceptable matches without rearranging her preference ranks of the men. It turns out, however, that sometimes agents in dynamic matching markets can manipulate the DA mechanism only by rearranging their preference ranking of the schools. Therefore, truncating or dropping strategies are not sufficient to identify whether the DA mechanism is manipulable in certain markets.

To overcome this computational problem, we construct a new algorithm. We first note that any two-year old student should reveal her true preferences because the DA mechanism is strategy-proof in static settings. In addition, if a one-year-old agent can manipulate the DA successfully by submitting a particular preference report, then all the preference reports that lead to the same matching must also be a beneficial manipulation for the agent. It turns out that in a typical market, there is only a limited number of matchings produced by the DA mechanism when an agent varies her preference reports. We use this result in the construction of our algorithm. Specifically, in each round our algorithm finds the DA matching corresponding to some report of a given one-year agent and disregards (nearly) all the preference reports that lead to the same DA matching. With our algorithm, we can significantly reduce the computational complexity of spanning every possible matching that a single agent can induce: to illustrate, we provide an example in which there are over 39 million strategy profiles that an agent might submit. Checking each such profile is infeasible, but our algorithm checks only 20 reports.

Using the algorithm described above, which we believe is of independent interest, we estimate the percentage of the markets in which a given agent can successfully manipulate the DA mechanism based on randomly generated data. We again consider the two priority systems. Furthermore, in order to capture the fact that schools differ in quality, we allow the preferences to be correlated. Under priorities satisfying guaranteed continued enrollment, the manipulation percentage is 0.99 percent when there are hundred schools with the capacity of one. This percentage drops to 0.08 percent if each school's capacity increases to 20 . Therefore, the manipulation percentage of the DA mechanism quickly converges to zero if the priorities satisfy IPA. On the other hand, under Danish priorities, the manipulation percentages are 31.4 percent to 37.73 percent, respectively, when there are hundred schools with capacities of 1 and 20 .

Our paper is related both to the literature on dynamic matching as well as the literature on matching in large markets. Kurino (2014), Pereyra (2013), Dur (2011), and Kennes, Monte, and Tumennasan (2014) study the centralized matching when the set of agents evolve in the overlapping generations fashion. Kurino (2014) focuses on the house allocation problem. Pereyra (2013) shows that the DA mechanism is stable and strategy-proof under seniority based priorities and time-invariant preferences. Dur (2011) studies the dynamic school choice problem faced by families with multiple children. Kennes, Monte, and Tumennasan (2014) considers the problem of allocating children to day care centers where each child attends day care centers in multiple periods and participating children evolve in the overlapping generations fashion. Both Dur (2011) and Kennes, Monte, and Tumennasan (2014) show that (i) the DA mechanism results in a stable matching and (ii) no mechanism is both stable and strategy-proof. The key reason for their impossibility 
results is that they consider a broader class of schools' priorities than the seniority based ones, due to the applications considered in these papers. Bloch and Cantala's (2013) dynamic matching problem is similar to ours, but they focus on the long-run properties of different assignment rules. Ünver (2010) studies the kidney exchange problem considering a dynamic environment in which the pool of agents evolves over time. Compte and Jehiel (2008) has a static model, but with a condition similar to our guaranteed continued enrollment.

We use the concept of stability for dynamic environments that was introduced by Kennes, Monte, and Tumennasan (2014). Other authors have proposed different stability notions, see, for example, Kurino (2008, 2009), Damiano and Lam (2005), Doval (2015), and Kadam and Kotowski (2018).

Our paper is also related to the literature on large matching markets, for which there is a broad recent literature. ${ }^{6}$ In static matching settings, Che and Kojima (2010) and Azevedo and Leshno (2016) consider the dynamics of the market size growth we study here: a large number of agents matched to a finite number of objects. Che and Kojima (2010) shows that the probabilistic serial dictatorship mechanism becomes strategy-proof as the number of agents along with the copies of the objects tend to infinity. Azevedo and Leshno (2016) considers the convergence of stable matchings in many-to-one matching settings as the market size increases, and they show that in a wide class of markets the stable matchings converge to a matching that is a unique stable matching in the continuum economy. We use some of Azevedo and Leshno's (2016) results extensively, but our paper differs from theirs in two major aspects: (i) our focus is the manipulation of the DA while theirs is stability in large or continuum economies, and (ii) we study dynamic environments while they concentrate on a static setting.

The paper is organized as follows. In Section I, we provide the model and the main definitions. In Section II, we describe a version of the deferred acceptance mechanism from Kennes, Monte, and Tumennasan (2014). In Section III, we examine the main properties of the mechanism in small economies. Section IV contains the results for an economy with a continuum of agents. In Section V, we prove our main convergence result. In Section VI, we provide our algorithm to check whether an agent can manipulate the DA mechanism, and we present our simulation results on the manipulability of the DA. The longer proofs are in the Appendix.

\section{Model}

Time $t$ is discrete and $t=1, \ldots, \infty$. There is a finite number of infinitely lived schools. ${ }^{7}$ Let $S=\left\{h, s_{1}, \ldots, s_{m}\right\}$ be the set of schools as well as the option of staying home, $h$. Let $\mathbf{r}=\left(r^{s}\right)_{s \in S}$ be the vector of capacities, with $r^{s} \in \mathbb{N}$.

\footnotetext{
${ }^{6}$ See Peranson and Roth (1999); Immorlica and Mahdian (2005); Kojima and Pathak (2009); Manea (2009); Che and Kojima (2010); Kojima and Manea (2010); Kojima, Pathak, and Roth (2013); Liu and Pycia (2016); Azevedo and Leshno (2016); Che and Tercieux (2018); Abdulkadiroğlu, Che, and Yasuda (2015); Azevedo and Hatfield (2013); Lee (2017); and Azevedo and Budish (forthcoming).

${ }^{7}$ Equivalently, one could think of daycare centers instead of schools, as in Kennes, Monte, and Tumennasan's (2014) prototypical application.
} 
We assume that each school other than home has a finite capacity, $r^{s}<\infty$, for all $s \neq h$, whereas home does not have a capacity constraint, that is, $r^{h}=\infty .8$

Each agent can attend school when she is one and two years old. ${ }^{9}$ If an agent attends schools $s$ and $s^{\prime}$ when she is 1 and 2, respectively, then we write $\left(s, s^{\prime}\right)$ to denote this.

An agent $i$ is one year old in period $t_{i}$ and has a strict preference relation $\succ_{i}$ over the set of possible pairs of schools. She is initially endowed with a priority score vector, $\mathbf{x}_{i}=\left(x_{i}^{S}\right)_{s \in S} \in[0,1)^{m+1}$. We represent each agent $i$ as a triplet $\left(t_{i}, \succ_{i}, \mathbf{x}_{i}\right)$ and write that $i=\left(t_{i}, \succ_{i}, \mathbf{x}_{i}\right)$.

At period $t \geq 1$, a finite set of one-year-old agents $I_{t}$ arrives, i.e., $i \in I_{t}$ if and only if $t_{i}=t$. We use the notation $I_{0}$ to denote the set of the agents who are two in period 1. Consequently, at any period $t \geq 1$, the set of school-age agents is $I_{t-1} \cup I_{t}$. The set of school-age agents evolves over time in an "overlapping generations" (OLG) fashion. Let $I=\left(I_{t}\right)_{t=0}^{\infty}$. With slight abuse of language, an economy is said to be finite if there is a finite number of agents in each period. Thus, a finite economy $E=(I, \mathbf{r}, X)$ specifies a finite set of agents per cohort, $I$, a vector of capacities, $\mathbf{r}$, and a priority updating rule, $X$, which will be defined later. We now define the matching in our setting. 10

DEFINITION 1 (Matching): A period-0 matching $\mu_{0}$ is a correspondence $\mu_{0}: I_{0} \cup S \rightarrow I_{0} \cup S$ such that $\mu_{0}(i)=\{h\}$ for all $i \in I_{0}, \mu_{0}(h)=I_{0}$ and $\mu_{0}(s)=\emptyset$ for all $s \neq h$.

A period- $t$ matching at any $t \geq 1, \mu_{t}$, is a correspondence $\mu_{t}: I_{t-1} \cup I_{t} \cup S \rightarrow I_{t-1} \cup I_{t} \cup S$ such that:

(i) for all $i \in I_{t-1} \cup I_{t},\left|\mu_{t}(i)\right|=1$, and $\mu_{t}(i) \subset S$;

(ii) for all $s \in S,\left|\mu_{t}(s)\right| \leq r^{s}$, and $\mu_{t}(s) \subset I_{t-1} \cup I_{t}$; ${ }^{11}$

(iii) for all $i \in I_{t-1} \cup I_{t}, i \in \mu_{t}(s)$ if and only if $s \in \mu_{t}(i)$.

A matching $\mu$ is a collection of period matchings: $\mu=\left(\mu_{t}\right)_{t=1}^{\infty}$.

With slight abuse of notation, denote by $\mu_{t}(i)$ the school to which agent $i$ is matched under $\mu_{t}$. We use the notation $\mu(i)$ to denote the pair of schools that $i$ is

\footnotetext{
${ }^{8}$ While we assume throughout the paper that capacities are fixed, our results are robust to increasing capacities over time (but not to decreasing ones).

${ }^{9}$ In the school choice setting with siblings, this assumption is equivalent to the one in which each family has two children. Incorporating families with one child into the model is straightforward (Dur 2011); in each period there is a set of agents who participate in the allocation process only once.

${ }^{10}$ Our definition of matching only specifies matchings that are observed. Other authors, including Kurino (2008) and Doval (2015), focus on full contingent plan of assignments.

${ }^{11}$ It can be that $\mu_{t}(s)=\emptyset$ for some school $s$. In such cases, no agent attends $s$ at period $t$.
} 
matched with under matching $\mu: \mu(i)=\left(\mu_{t_{i}}(i), \mu_{t_{i}+1}(i)\right)$. Let $\mathcal{M}_{t}$ be the set of period $t$ matchings.

From the definition above, observe that in period 0, every agent stays home, i.e., the schools start their operation at period 1 . As a consequence of this assumption, all matchings we consider have a common period 0 matching in which all school-age agents are matched with $h \cdot{ }^{12}$

Agents' Preferences.-Each agent $i$ has strict preferences, $\succ_{i}$. We write $\left(s, s^{\prime}\right) \succeq_{i}\left(\bar{s}, \bar{s}^{\prime}\right)$ if either $\left(s, s^{\prime}\right) \succ_{i}\left(\bar{s}, \bar{s}^{\prime}\right)$ or $\left(s, s^{\prime}\right)=\left(\bar{s}, \bar{s}^{\prime}\right)$. We impose some restrictions on agents' preferences. First, we assume that each agent has an underlying ranking over schools (not the pairs of schools) that stays stable over time. ${ }^{13}$ Consequently, if a school $s$ is superior to another school $s^{\prime}$, then it must be that $(s, s) \succ_{i}\left(s^{\prime}, s^{\prime}\right)$. In addition, there is no complementarity from attending two different schools, but there could be from attending the same school for two periods. Specifically, attending an inferior school $s^{\prime}$ in one period and a different school $s^{\prime \prime}$ in the other eligible period is always worse than attending a superior school $s$ and school $s^{\prime \prime}$. On the other hand, attending $s^{\prime}$ for two periods may be better than attending the superior school $s$ in one period and $s^{\prime}$ in the other eligible period. This latter scenario could be due to switching costs. We collect these assumptions below.

ASSUMPTION 1 (Rankability): If $(s, s) \succ_{i}\left(s^{\prime}, s^{\prime}\right)$ for some $i$, $s$, and $s^{\prime}$, then $\left(s, s^{\prime \prime}\right) \succ_{i}\left(s^{\prime}, s^{\prime \prime}\right)$ and $\left(s^{\prime \prime}, s\right) \succ_{i}\left(s^{\prime \prime}, s^{\prime}\right)$ for any $s^{\prime \prime} \neq s^{\prime}$. Let $\mathcal{R}$ be the set of preferences satisfying Assumption 1.

Now let us define a stronger version of the rankability assumption which rules out the possibility that attending an inferior school for two periods is better than attending this school for one period and a superior school in the other eligible period.

DEFINITION 2 (Strong Rankability): If $(s, s) \succ_{i}\left(s^{\prime}, s^{\prime}\right)$ for some $i, s$, and $s^{\prime}$, then $\left(s, s^{\prime \prime}\right) \succ_{i}\left(s^{\prime}, s^{\prime \prime}\right)$ and $\left(s^{\prime \prime}, s\right) \succ_{i}\left(s^{\prime \prime}, s^{\prime}\right)$ for any $s^{\prime \prime}$.

The strong rankability assumption means that the switching costs are not too large. We remark here that the sole purpose of the strong rankability assumption is to simplify the presentation of some of our examples, i.e., none of our theoretical results rely on this stronger assumption.

When defining the preferences, we are following a more general axiomatic approach. Before proceeding further, let us give an example that illustrates a parametric approach.

\footnotetext{
${ }^{12}$ We can relax this restriction so that a period 0 matching is defined similarly to the other period matchings. Given that we interpret period 0 as the period that occurred right before the start of our model, period 0 matching cannot be altered. Thus, all matchings must have a common period 0 matching, which is one of the primitives of the model. With the modified definition of a period 0 matching, all the results except those in Section V go through. In footnote 25, we will present an additional assumption that guarantees the validity of results in Section V.

${ }^{13}$ In the school choice with siblings setting this means that two children of the same household have the same preferences of schools.
} 
Example 3 (Parametric Utility Function): Suppose that by attending school $s$ for one period, child $i$ benefits $u_{i}(s)>0$, which does not depend on the child's age. Each child weights the periods by $(1-\delta)>0$ in the period when she is born, $t_{i}$ and $\delta>0$, when she is in the market for the second time. Note that the case where $\delta<1$ is the typical time-discounting framework if we normalize period 1 and consider a discount rate $\beta=\frac{\delta}{1-\delta}$. Moreover, child $i$ incurs a switching cost of $c_{i}>0$, that is, a cost incurred only if there was a change in the school from period $t$ to period $t+1$. Finally, the utility of child $i$ attending schools $s$ and $s^{\prime}$ at her respective ages of 1 and 2 is

$$
U_{i}\left(s, s^{\prime}\right)= \begin{cases}(1-\delta) u_{i}(s)+\delta u_{i}\left(s^{\prime}\right)-c_{i} & \text { if } s \neq s^{\prime} \text { and } s \neq h \\ (1-\delta) u_{i}(s)+\delta u_{i}\left(s^{\prime}\right) & \text { otherwise }\end{cases}
$$

Clearly, these underlying preferences for the children satisfy Assumption 1 and furthermore, they satisfy strong rankability as well if the cost $c_{i}$ of a school to school change is sufficiently small.

Schools' Priorities and Priority Updating Rules.-We previously mentioned that each agent $i$ is endowed with a priority score vector $\mathbf{x}_{i}$. This priority score vector will be used to determine the agent's priority in each school in the period that this agent is one year old, i.e., in period $t_{i}$. It is fixed at the agent's birth year, but may change in the following period when the schools' priorities are history-dependent, which we will assume. If an agent $i$ has a priority score vector $\mathbf{x}_{i}$ and an agent $j$, born in the same period has a priority score vector given by $\mathbf{x}_{j}$, with $x_{i}^{s}>x_{j}^{s}$, for some $s \in S$, we have that agent $i$ has a higher priority in period $t_{i}$ than agent $j$ at school $s .14$

ASSUMPTION 2 (Strict Priorities): For any two agents $i, j$ in $I_{t-1} \cup I_{t}, x_{i}^{s} \neq x_{j}^{s}$ for all $s \in S$.

Given the dynamic nature of our problem, we will consider the case in which the priority score of agent $i$ at period $t_{i}+1$ depends on the previous period's matching. We consider only two specific channels through which the priority vector of an agent may change over time. This is motivated by natural applications of dynamic matching problems, such as the centralized assignment of young children to public day care centers, the assignment of teachers to public schools, and when there is priority for incumbent students and their siblings in the school choice problem.

The two priority updating rules that we consider are: (i) the schools give the highest priorities to their previously enrolled agents and this is the only way in which priorities change over time; and (ii) additionally to giving highest priority to their previous attendees, schools also give high priority to agents who were not enrolled in any school in the previous period despite their eligibility. The high priority to

\footnotetext{
${ }^{14}$ We should note that in many applications priorities are coarse and a tie-breaking rule is constructed to determine the priority ordering of the students. The assumptions in this paper imply that the same tie-breaking rule is used across periods.
} 
previously enrolled agents is a feature present in many real-life applications. Given the importance of this restriction for many different systems and on its natural appeal, i.e., agents will not be forced out of a school, we will maintain this assumption throughout our paper. That is, both priority systems that we consider satisfy this condition. The second priority system satisfies both channels, where the second channel is motivated by a concept of the assignment of young children to day care centers in Denmark, called guaranteed spot. We return to this in Assumption 3b.

To formally incorporate the history-dependence of priorities in our model, we define the priority score function, or priority updating rule, of each agent $i$ at some school $s$ as a mapping $X_{i}^{s}: \mathcal{M}_{t_{i}-1} \cup \mathcal{M}_{t_{i}} \rightarrow[0,2]$, such that $X_{i}^{s}\left(\mu_{t_{i}-1}\right)=x_{i}^{s}$ for all $\mu_{t_{i}-1} \in \mathcal{M}_{t_{i}-1}$. This means that if a period- $t$ matching was $\mu_{t} \in \mathcal{M}_{t}$, then at period $t+1$ the priority score of a school-age agent $i$ at school $s$ is $X_{i}^{s}\left(\mu_{t}\right) \cdot{ }^{15}$ If $i$ was born in period $t+1$, then her priority score at school $s$ must be $X_{i}^{s}\left(\mu_{t}\right)=x_{i}^{s}$, which is exogenously determined.

For a given matching $\mu_{t} \in \mathcal{M}_{t}$, we denote the priority score vector of $s$ at period $t+1$ by $X^{s}\left(\mu_{t}\right) \equiv\left(X_{i}^{s}\left(\mu_{t}\right)\right)_{i \in I_{t} \cup I_{t+1}}$ and school-age agent $i$ 's priority scores at all schools by $X_{i}\left(\mu_{t}\right) \equiv\left(X_{i}^{s}\left(\mu_{t}\right)\right)_{s \in S}$. We will maintain the following assumption throughout the paper.

ASSUMPTION 3 (Gauranteed Continued Enrollment): Each agent's priority score function at any school sfor which $i \in \mu_{t_{i}}(s)$ satisfies

$$
X_{i}^{s}\left(\mu_{t_{i}}\right)>X_{j}^{s}\left(\mu_{t_{i}}\right), \quad \forall j \notin \mu_{t_{i}}(s) .
$$

This assumption states that an agent who is matched to a school $s$ when she is one year old will have the highest priority score at that school in the subsequent year. In addition, the agent's priority score at any other school remains the same unless she was matched to that school at the age of one.

The first class of economies that we consider are such that the priority score function is history-dependent only through the guaranteed continued enrollment. When this is the case, we will say that the priority system satisfies independence of past attendances, or simply IPA.

ASSUMPTION 3a (Independence of Past Attendances (IPA)): Each agent's priority score function at any school s satisfies that

$$
X_{i}^{s}\left(\mu_{t_{i}}\right)= \begin{cases}2 & \text { if } i \in \mu_{t_{i}}(s) \\ x_{i}^{s} & \text { otherwise }\end{cases}
$$

for all $\mu_{t_{i}} \in M_{t_{i}}$

\footnotetext{
${ }^{15}$ Moving forward it is convenient to have one notation that expresses the priority scores of both oneand two-year-old agents.
} 
Here, observe that the attendees of any school $s$ at some period $t$ will have the same priority score of 2 at the school in the following period, where note that $2>x_{i}^{s} \forall i, s .16$

In the current Danish day care assignment system IPA is not satisfied; if an older child who has not attended any day care previously asks for a guaranteed spot then at some day care she is given a priority over all the children who have attended some day care previously, as well as some younger children who are participating in the assignment process for the first time. ${ }^{17}$ The current rule does not spell out for which day care this older child gets a priority. ${ }^{18}$ Due to incompleteness of this rule, we cannot accurately define the current Danish priority system. However, given its practical importance we would like to examine this feature of the current Danish priority system closely when we study the incentives to manipulate the deferred acceptance mechanism. Consequently, we will consider throughout the paper a second priority system in which each school assigns a higher priority to two-year-old children who have not attended any school in the previous period over one-year-old children and over two-year-old children who previously attended a school other than the one in question. ${ }^{19}$ That is, we maintain the guaranteed continued enrollment, but also add the feature that children who have not attended any school have higher priority over other children who attended some school.

ASSUMPTION 3b (Failure of IPA: Danish Priorities): A priority scoring system is Danish if each agent's priority score function at each school s satisfies the following condition:

$$
X_{i}^{s}\left(\mu_{t_{i}}\right)= \begin{cases}2 & \text { if } i \in \mu_{t_{i}}(s) \\ 1+x_{i}^{S} & \text { if } i \in \mu_{t_{i}}(h) \\ x_{i}^{s} & \text { otherwise }\end{cases}
$$

for all $\mu_{t_{i}} \in M_{t_{i}}$

In the Danish priority scoring system an agent who stays at home when she is young will have a priority score of $1+x_{i}^{s}$ at school $s$ in the following period. Consequently, by staying home at age one, an agent jumps ahead of almost all agents (except the school's previous period's attendees) in the priority ranking of any school at age two. However, observe here that the relative rankings of those who stay home when they are age one do not change.

\footnotetext{
${ }^{16}$ This assumption, as we will see later, does not cause any problem running the version of the deferred acceptance algorithm used in this paper-note that given Assumption 2, we will never have the problem that there are more students with the same score at a school than the school's capacity.

${ }^{17}$ Children who have special needs (due to disability or due to a foreign language spoken at home) or who have siblings at a specific daycare always have higher priority over the children who ask for a guaranteed spot.

${ }^{18}$ Officials in charge of the assignments decide this on a case-by-case basis.

${ }^{19}$ This assumption is stronger than what is actually done in practice, however, it illustrates the issue well.
} 
It is important to note that a priority system might satisfy Assumption 3a or Assumption 3b, but not both. Also note that both Assumptions 3a and 3b respect the guaranteed continued enrollment assumption.

Threshold Scores.-For a given matching $\mu$, let the period- $t$ threshold score of school $s$ corresponding to $\mu$ be $p_{t}^{s}$ such that

$$
p_{t}^{s}= \begin{cases}0 & \text { if }\left|\mu_{t}(s)\right|<r^{s} \\ \inf _{i \in \mu_{t}(s)} X_{i}^{s}\left(\mu_{t-1}\right) & \text { otherwise }\end{cases}
$$

Observe here that the threshold score of $h$ corresponding to any matching is always 0 because $h$ does not have any capacity restriction. We use the following notations: $\mathbf{p}_{t}=\left(p_{t}^{s}\right)_{s \in S}$ and $\mathbf{p}=\left(\mathbf{p}_{t}\right)_{t=1}^{\infty}$.

\section{Mechanism and the Deferred Acceptance}

A mechanism (for finite economies) is a systematic process that assigns a matching for each finite economy. We use the notation $\varphi$ to denote a typical mechanism for finite economies. Let $\varphi_{i}(E)$ be the pair of schools to which agent $i$ is matched under $\varphi$. For each mechanism, there is an associated preference revelation game. If no agent has incentives to misrepresent her preferences in this game, then we say the mechanism is strategy-proof. Below we state the formal definition.

DEFINITION 3 (Strategy-Proofness): We say that a mechanism $\varphi$ is manipulable (individually) at a finite economy $E$ if there exists an economy $E^{\prime}=\left(I^{\prime}, \mathbf{r}, X\right)$, and an agent $i \in I$, such that:

(i) $E^{\prime}$ differs from $E$ only in agent $i$ 's preference ordering (i.e., $\succ_{i} \neq \succ_{i}^{\prime}$ and $\succ_{j}=\succ_{j}^{\prime}$, for all $j \neq i$ ). and

(ii) $\varphi_{i}\left(E^{\prime}\right) \succ_{i} \varphi_{i}(E)$.

A mechanism $\varphi$ is strategy-proof if it is not manipulable for any finite economy.

The DA is widely used in school choice problems, so it is natural to ask how it performs in a dynamic matching problem. A first technical difficulty is that the DA is framed as each agent reporting her preference profile ranking the schools-not the pairs of schools. Since our goal is to study the DA, as it is known and used in practice, we will present a version of the DA for multiple periods (which is a DA period-by-period).

Before we define this mechanism in detail, let us present a concept that we will call isolated preferences (Kennes, Monte, and Tumennasan 2014). 20

\footnotetext{
${ }^{20}$ Consider period $t \geq 1$, and suppose that the period matchings up until this period are assigned. Let us now assume that the agents who are eligible to attend school in this period are asked to rank the schools (not pairs
} 
DEFINITION 4 (Isolated Preference Relation): For any given period $t \geq 1$, and for a given matching $\mu_{t-1}$, the isolated preference relation of period $t, P_{i}\left(\mu_{t-1}\right)$ is a binary relation satisfying:

(i) $\forall i \in I_{t}: s P_{i}\left(\mu_{t-1}\right) s^{\prime}$ if and only if $(s, s) \succ_{i}\left(s^{\prime}, s^{\prime}\right)$ for any $s, s^{\prime} \in S$;

(ii) $\forall i \in I_{t-1}: s P_{i}\left(\mu_{t-1}\right) s^{\prime}$ if and only if $\left(\mu_{t-1}(i), s\right) \succ_{i}\left(\mu_{t-1}(i), s^{\prime}\right)$ for any $s, s^{\prime} \in S$.

We here note that in any period the isolated preferences of one-year-old agents do not depend on the preceding period's matching. Let $P\left(\mu_{t-1}\right)$ be the collection of isolated preferences for the school-age agents in period $t$, i.e., $P\left(\mu_{t-1}\right) \equiv\left(P_{i}\left(\mu_{t-1}\right)\right)_{i \in I_{t-1} \cup I_{t}}$. In addition, the notation $\mathcal{P}$ denotes the set of all possible isolated preferences. We will usually write $P_{i}$ instead of $P_{i}\left(\mu_{t-1}\right)$ as long as doing so does not create confusion.

We note here that if an agent's preferences are strongly rankable, then her isolated preferences will be the same regardless of her age or the previous period's matching.

The period-by-period Gale and Shapley deferred acceptance mechanism will utilize the isolated preferences. Kennes, Monte, and Tumennasan (2014) consider this mechanism and denote it by Deferred Acceptance Mechanism using Isolated Preferences (DA-IP). In this mechanism agents report their full preference profile and the mechanism constructs the isolated preferences. (Alternatively one could define it as a mechanism in which the school-age agents in any given period report their isolated preferences over schools knowing their previous period's matchings). Formally, the DA-IP mechanism associates each economy with the matching that is the result of the DA-IP algorithm, which we define below.

Fix a finite economy $E$. Because this paper revolves around the DA-IP mechanism, we reserve the notation $\eta$ for the matching that is the result of the DA-IP algorithm. Recall that for all matchings, every agent stays home in period 0. Thus, every agent is assigned $h$ at $\eta_{0}$. The DA-IP algorithm determines period-1 DA-IP matching $\eta_{1}$ using $\eta_{0}$. Once the period-1 DA-IP matching is determined, the algorithm uses this matching to determine the period-2 DA-IP matching $\eta_{2}$, and it does so for every subsequent period. The period-1 DA-IP matching is found by running the following algorithm in finite rounds (in essence, the well-known deferred acceptance algorithm).

Period-1 Assignment: Set the isolated preferences for each school-age agent $i$ in this period to $P_{i}\left(\eta_{0}\right)$. In addition, set the priority score vector of each school $s$ in this period to $X^{s}\left(\eta_{0}\right)$.

of schools). Given the motivations for the rankability assumption, perhaps any one-year-old agent $i$ would rank $s$ over $s^{\prime}$ if and only if $s$ is superior to $s^{\prime}$, i.e., if and only if $(s, s) \succ_{i}\left(s^{\prime}, s^{\prime}\right)$. A two year old, on the other hand, would rank $s$ over $s^{\prime}$ if and only if $\left(\mu_{t}(i), s\right) \succ_{i}\left(\mu_{t}(i), s^{\prime}\right)$, given that she knows her match in the previous period. This is the motivation behind the concept of isolated preferences. 
Round 1: Each school-age agent of period 1 applies to her most preferred school according to her isolated preferences $\left(P_{i}\left(\eta_{0}\right)\right.$ in this case). Each school $s$ then "holds" the $r_{s}$ applicants with the highest priority score (according to $X^{s}\left(\eta_{0}\right)$ in this case) and rejects all others.

In general, at:

Round $k$ : each agent whose application was rejected in the previous round applies to her most preferred school (according to her isolated preferences, $P_{i}\left(\eta_{0}\right)$ ) that has not rejected her. Each school $s$ considers the pool of applicants composed of the new applicants and the agents whom $s$ has been holding from the previous round. Each school $s$ then "holds" the $r_{s}$ agents in the pool who have the highest priority score (according to $X^{s}\left(\eta_{0}\right)$ in this case) and rejects all others.

The algorithm terminates when no proposal is rejected and each agent is assigned her final tentative assignment. This final matching is $\eta_{1}$.

Period-2 Assignment: Set the isolated preferences of each school-age $i$ in this period to $P_{i}\left(\eta_{1}\right)$ and the priority score vector of each school $s$ in this period to $X^{s}\left(\eta_{1}\right)$. Now using the algorithm described above, we can find the period-2 DA-IP matching $\eta_{2}$.

Period- $t$ Assignment: Set the isolated preferences of each school-age $i$ in this period to $P_{i}\left(\eta_{t-1}\right)$ and the priority score vector of each school $s$ in this period to $X^{s}\left(\eta_{t-1}\right)$. Now using the algorithm described above, we can find the period- $t$ DA-IP matching $\eta_{t}$.

As we mentioned at the outset of this paper, stability is a key reason why the DA plays a prominent role in the static school choice problem. In fact, its adoption in dynamic settings-DA-IP_-also turns out to be stable as long as the priorities satisfy IPA (Theorem 1 of Kennes, Monte, and Tumennasan 2014). ${ }^{21}$

Many real-life mechanisms allow agents to submit different reports in different periods. For instance, in the Danish day care system, any agent who is enrolled at a day care currently can submit a new ranking over day cares. The same is true for the teacher allocation problems. Observe that the DA-IP can handle such cases because it runs on a period-by-period basis. Consequently, we can allow agents to report two preference reports in different periods. ${ }^{22}$ Then the period DA-IP mechanisms only consider the reports of that period. For those who are reporting truthfully, reporting

\footnotetext{
${ }^{21}$ We are considering here the stability notion introduced by Kennes, Monte, and Tumennasan (2014) that captures justified envyness in a dynamic environment, where priorities are changing over time as a function of previous allocations. Other definitions prior to ours include Kurino's (2008) notion of dynamic pairwise-stability, Damiano and Lam's (2005) self-sustaining stability, and, more recently, Doval (2018) and Kadam and Kotowski (2018) also provide their own definition of dynamic stability.

${ }^{22}$ Both or one of them can be replaced by isolated preference reports. We believe that a two-year-old ranking schools is not controversial because she knows her match of the previous period. In this sense, the ranking is the isolated preferences for two-year-olds. We believe that the isolated preferences are natural rankings over schools even for one-year-olds.
} 
the preference once or twice does not matter. However, reporting one's preferences twice of course creates new possibilities of manipulation. For our theoretical results, whether we allow one or two preference reports makes no difference. However, they do for simulation results. The numbers we report correspond to the situation in which the agents report their preferences twice.

\section{Manipulation in Small Economies}

It is well known that in static settings, the agent proposing DA mechanism is strategy-proof. In contrast, Kennes, Monte, and Tumennasan (2014) show that in dynamic environments the DA-IP mechanism is not strategy-proof, ${ }^{23}$ i.e., in some small economies an agent finds it profitable to misrepresent her preferences (when everyone else reports her preferences truthfully).

THEOREM 1 (DA-IP is Manipulable): The DA-IP mechanism is not strategy proof.

In the introduction we presented an example (Example 1) in which an agent manipulates her report and is assigned to a worse school in the first period, but to a better school in the second period. Overall her payoff improved. This example is enough to prove the theorem above. Below we present another example (different from Example 1 and from the one that Kennes, Monte, and Tumennasan 2014 use in their proof of the impossibility result) to show that there is also a class of manipulations in which agents do not have to get worse off in their first period in order to manipulate the DA-IP, i.e., a manipulating agent gets to be assigned to the same school that she would get assigned under truth-telling. This, of course, follows from the fact that the DA is bossy.

Example 4 (Manipulation under Assumption 3a): Consider the following economy $E$ with five schools $s_{1}, s_{2}, s_{3}, s_{4}$, and $s_{5}$, and six agents, $i_{1}, i_{2}, i_{3}, i_{4}, i_{5}$, and $i_{6}$. Each school has a capacity of one agent and suppose that $I_{0}=\left\{i_{1}, i_{2}\right\}$, $I_{1}=\left\{i_{3}, i_{4}, i_{5}\right\}, I_{2}=\left\{i_{6}\right\}$.

Each agent's preferences are strongly rankable, and the agents' isolated preferences are as follows:

$\begin{array}{llllll}i_{1}: & s_{1} & & & & \\ i_{2}: & s_{2} & & & & \\ i_{3}: & s_{1} & s_{5} & s_{3} & s_{4} & s_{2} \\ i_{4}: & s_{4} & s_{2} & s_{3} & s_{5} & s_{1} \\ i_{5}: & s_{3} & s_{1} & s_{4} & s_{5} & s_{2} \\ i_{6}: & s_{4} & s_{1} & s_{3} & s_{5} & s_{2}\end{array}$

${ }^{23}$ Their result is even stronger: no stable and strategy-proof mechanism exists. 
The priority scores of the agents are presented below, where each line shows how each different school ranks the different students:

$$
\begin{aligned}
& x_{i_{1}}^{s_{1}}>x_{i_{6}}^{s_{1}}>x_{i_{3}}^{s_{1}}>x_{i_{4}}^{s_{1}}>x_{i_{5}}^{s_{1}}>x_{i_{2}}^{s_{1}} \\
& x_{i_{2}}^{s_{2}}>x_{i_{4}}^{s_{2}}>x_{i_{1}}^{s_{2}}>x_{i_{3}}^{s_{2}}>x_{i_{5}}^{s_{2}}>x_{i_{6}}^{s_{2}} \\
& x_{i_{6}}^{s_{3}}>x_{i_{4}}^{s_{3}}>x_{i_{3}}^{s_{3}}>x_{i_{5}}^{s_{3}}>x_{i_{1}}^{s_{3}}>x_{i_{2}}^{s_{3}} . \\
& x_{i_{6}}^{s_{4}}>x_{i_{5}}^{s_{4}}>x_{i_{3}}^{s_{4}}>x_{i_{4}}^{s_{4}}>x_{i_{1}}^{s_{4}}>x_{i_{2}}^{s_{4}} \\
& x_{i_{3}}^{s_{5}}>x_{i_{5}}^{s_{5}}>x_{i_{4}}^{s_{5}}>x_{i_{2}}^{s_{5}}>x_{i_{1}}^{s_{5}}>x_{i_{6}}^{s_{5}}
\end{aligned}
$$

Let $E^{\prime}$ be an economy which differs from $E$ only in agent $i_{3}$ 's preferences, which are still strongly rankable and given by

$$
i_{3}: \quad s_{1} \quad s_{3} s_{5}
$$

The DA-IP matchings in economies $E$ and $E^{\prime}$ are given

\section{Economy $E$}

Period $1 \quad\left(\begin{array}{llllll}i_{1} & i_{2} & i_{3} & i_{4} & i_{5} & - \\ s_{1} & s_{2} & s_{5} & s_{4} & s_{3} & -\end{array}\right)$

Period $2\left(\begin{array}{llllll}- & - & i_{3} & i_{4} & i_{5} & i_{6} \\ - & - & s_{5} & s_{4} & s_{3} & s_{1}\end{array}\right)$

\section{Economy $E^{\prime}$}

Period $1 \quad\left(\begin{array}{llllll}i_{1} & i_{2} & i_{3} & i_{4} & i_{5} & - \\ s_{1} & s_{2} & s_{5} & s_{3} & s_{4} & -\end{array}\right)$.

Period $2\left(\begin{array}{llllll}- & - & i_{3} & i_{4} & i_{5} & i_{6} \\ - & - & s_{1} & s_{2} & s_{3} & s_{4}\end{array}\right)$

Thus, agent $i_{3}$ has an incentive to manipulate the DA-IP mechanism at economy $E$. Interestingly, agent $i_{3}$ still gets to match with school $s_{5}$ in period 1 but gets to match with a better school, $s_{1}$, in period 2 .

In the above example, agent $i_{3}$ manipulates the DA-IP successfully by ranking $s_{3}$ ahead of $s_{5}$, and there is no other preference report that improves $i_{3}$ over truth telling. In particular, there is a bossy manipulation, but no nonbossy manipulation. Agents must alter the relative ranking of the schools in order to successfully manipulate the DA-IP mechanism.

Observe from the Examples 1 and 4 that at her successful manipulation the agent does not get better when she is age 1 but gets better when she is age 2 . This turns out to be a general phenomenon as shown in Lemma 1.

LEMMA 1: If an agent $i$ can successfully manipulate the DA-IP mechanism in a finite economy $E$, then $i$ cannot be born in period 0 . In addition, if the DA-IP matchings in economy $E$ and at i's successful manipulation are $\eta$ and $\hat{\eta}$,respectively, then the following conditions must be satisfied:

$$
\left(\hat{\eta}_{t_{i}+1}(i), \hat{\eta}_{t_{i}+1}(i)\right) \succ_{i}\left(\eta_{t_{i}+1}(i), \eta_{t_{i}+1}(i)\right) \succeq_{i}\left(\eta_{t_{i}}(i), \eta_{t_{i}}(i)\right) \succeq_{i}\left(\hat{\eta}_{t_{i}}(i), \hat{\eta}_{t_{i}}(i)\right)
$$




\section{PROOF:}

See Appendix C.

This lemma shows that to manipulate the DA-IP mechanism successfully, one will have to accept a weakly worse allocation when she is young in order to improve her future assignment. This is indeed true even in the Danish priority system for which $I P A$ is violated. However, there is an essential difference in the information required for manipulation. Recall that Example 2 in the introduction presented an example of a system with the Danish priority structure in which the DA-IP is manipulated.

Examples 1 and 4, on one hand, and Example 2, on the other hand, suggest that successful manipulations for different priority systems differ in terms of required "sophistication." In Example 2, the Danish system, the agent could manipulate simply by staying home when she is young; by doing that, she jumps ahead of almost everyone in the priorities of all schools. This is a relatively simple manipulation as it only involves one action, which is staying home when young-which ultimately improves the agent's priority score relative to the scores of others. On the other hand, manipulating the DA-IP mechanism in systems satisfying IPA is rather difficult. To see this let us return to Example 4 . When agent $i_{3}$ misreports her preferences, the agent who was matched to school $s_{1}$ in period $t+1$ under truth telling (in our case $i_{6}$ ) will still have priority over $i_{3}$ at school $s_{1}$. In other words, agent $i_{3}$ 's priority score at $s_{1}$ does not improve at all no matter what she does. This means that agent $i_{3}$ 's manipulation must benefit agent $i_{6}$ so that she never applies to school $s_{1}$. This, of course, is possible in the example we considered, but the agent must be rather sophisticated to see through all of these possible effects of her manipulation.

\section{School Assignment with a Continuum of Agents}

In this section, we study the school assignment problem when there is a finite number of schools and a continuum of agents. Recall that throughout the paper we will assume guaranteed continued enrollment (Assumption 3). We will compare the strategic performance of the DA-IP under the different priority structures considered in the previous section, namely Assumptions 3a and $3 \mathrm{~b}$. An economy with a continuum of agents provides a clean environment to obtain two important results for our paper: (i) that under IPA the DA-IP is non-manipulable in the continuum economy, and that (ii) under Danish priorities the DA-IP remains manipulable even in a continuum economy. Given the positive result of the DA-IP implementation in a continuum economy under IPA, we study in the next section the convergence of large markets to a continuum market; and in Section VI, we provide simulation results to study large, but finite markets, and again we compare the strategic performance of the DA-IP under the to different priority structures.

To study the continuum economy in a dynamic environment we borrow some of the notation from Azevedo and Leshno (2016) and adapt some of their results to our dynamic environment. In this part, we again assume that each agent is endowed with a strict preference ordering over the schools and a vector of threshold scores $\mathbf{x}$. The set of schools is finite, but we assume that the set of agents born in each period 
$t, \bar{I}_{t}=t \times \mathcal{R} \times[0,1)^{m+1}$, is a continuum mass of students, where recall $\mathcal{R}$ is the set of strict preference orderings satisfying rankability. The set of children is thus $\bar{I}=\cup_{t=0}^{\infty} \bar{I}_{t}=\mathbb{N} \times \mathcal{R} \times[0,1)^{m+1}$. Let $\bar{\nu}$ be a measure on $\bar{I}$. We assume that the distribution of the children born in each period is identical, i.e., for any $t \neq \tau, \bar{J}_{t} \subseteq \bar{I}_{t}$ and $\bar{J}_{\tau} \subseteq \bar{I}_{\tau}$, such that child $(t, \succ, \mathbf{x}) \in \bar{J}_{t}$ if and only if child $(\tau, \succ, \mathbf{x}) \in \bar{J}_{\tau}$, we have that $\bar{\nu}\left(\bar{J}_{t}\right)=\bar{\nu}\left(\bar{J}_{\tau}\right)$. One can relax this assumption without affecting the main results of the paper, but the notation will be considerably more complicated. Let $\overline{\mathbf{r}}$ be the vector of capacities. A continuum economy is $\bar{F}=(\bar{\nu}, \overline{\mathbf{r}}, \bar{X})$, with $\bar{X}$ being the score updating rule, as before. For simplicity, we assume that $\bar{\nu}\left(I_{t}\right)=1$, for all $t \geq 0$. We will maintain the following assumption throughout the paper.

ASSUMPTION 4 (Strict Priorities): For any school $s \in S$, the measure of agents born in the same period who have the same priority at this school is 0, i.e., $\bar{\nu}\left(\left\{i: t_{i}=t\right.\right.$ and $\left.\left.x_{i}^{s}=e\right\}\right)=0$ for any $t \geq 0$ and $\forall e \in[0,1)$.

The assumption above immediately implies that the measure of each agent is 0 , i.e., $\bar{\nu}(\{i\})=0$.

We are now ready to present the definition of matching which, as in finite economies, is a collection of period matchings. The definition of a period matching is the same one that Azevedo and Leshno (2016) use in static continuum economies.

DEFINITION 5 (Matching): A period-0 matching $\bar{\mu}_{0}$ is a function $\bar{\mu}_{0}: \bar{I}_{0} \cup S \rightarrow \bar{I}_{0} \cup S$, such that $\left|\bar{\mu}_{0}(i)\right|=1$ and $\bar{\mu}_{0}(i)=\{h\}$, for all $i \in I_{0}, \bar{\mu}_{0}(h)=I_{0}$ and $\bar{\mu}_{0}(s)=\emptyset$, for all $s \neq h$.

A period matching in period $t \geq 1, \quad \bar{\mu}_{t}, \quad$ is a function $\bar{\mu}^{t}: \bar{I}_{t} \cup \bar{I}_{t-1} \cup S \rightarrow \bar{I}_{t} \cup \bar{I}_{t-1} \cup S$ such that:

(i) for all $i \in \bar{I}_{t-1} \cup \bar{I}_{t},\left|\bar{\mu}_{t}(i)\right|=1$ and $\bar{\mu}_{t}(i) \in S$;

(ii) for all $s \in S, \bar{\nu}\left(\bar{I}_{t-1} \cap \bar{\mu}_{t}(s)\right)+\bar{\nu}\left(\bar{I}_{t} \cap \bar{\mu}_{t}(s)\right) \leq \bar{r}^{s}$ and $\bar{\mu}_{t}(s) \subset \bar{I}_{t-1} \cup \bar{I}_{t}$;

(iii) for all $i \in \bar{I}_{t-1} \cup \bar{I}_{t}, i \in \bar{\mu}_{t}(s)$ if and only if $s=\bar{\mu}_{t}(i)$.

(iv) Each period-t matching is right continuous, i.e., for any sequence of agents $\left\{i^{k}\right\}=\left\{\left(\tau, \succ, x^{k}\right)\right\}$ where $\tau=t-1$, t converging to $i=(\tau, \succ, x)$, we can find some large $K$ such that $\bar{\mu}_{t}\left(i^{k}\right)=\bar{\mu}_{t}(i)$ for all $k>K$.

A matching $\bar{\mu}$ is a collection of period matchings: $\bar{\mu}=\left(\bar{\mu}_{0}, \bar{\mu}_{1}, \ldots, \bar{\mu}_{t}, \ldots\right)$.

Requirement (iv) rules out a multiplicity of stable matchings that differ only by sets of measure zero. As in the finite economy case, we assume 
that in period 0 everyone stays home. We use the following notations: $\bar{\nu}\left(\bar{\mu}_{t}(s)\right) \equiv \bar{\nu}\left(\bar{I}_{t-1} \cap \bar{\mu}_{t}(s)\right)+\bar{\nu}\left(\bar{I}_{t} \cap \bar{\mu}_{t}(s)\right)$ and $\bar{\mu}(i) \equiv\left(\bar{\mu}_{t_{i}}(i), \bar{\mu}_{t_{i}+1}(i)\right)$.

Now that we have defined matching in continuum economies, we can define the isolated preferences $\left(\bar{P}_{i}\left(\bar{\mu}_{t}\right)\right)$, priority score functions $\left(\bar{X}_{i}^{s}(\bar{\mu})\right)$, threshold scores $\left(\bar{p}^{s}(\bar{\mu})\right)$, and mechanism $(\bar{\varphi})$ for continuum economies as we did in finite economies. Furthermore, all the assumptions and notions used in finite economies such as IPA, the Danish priority system, stability, manipulability, and strategy-proofness are analogously defined for continuum economies.

The DA-IP Mechanism in Continuum Economies.-The DA-IP mechanism for continuum economies associates each continuum economy with the matching that is the result of the DA-IP algorithm, which we define below.

Fix a continuum economy $\bar{F}$. We reserve the notation $\bar{\eta}$ for the matching which is the result of the DA-IP algorithm. By the definition of matching, it must be that $\bar{\eta}_{0}$ matches each agent in $\bar{I}_{0}$ to $h$. Now the period-1 DA-IP matching $\bar{\eta}_{1}$ is found by running the following algorithm: ${ }^{24}$

Set the isolated preferences for each school-age agent $i$ in this period to $\bar{P}_{i}\left(\eta_{0}\right)$. In addition, set the priority score vector of each school $s$ in this period to $\bar{X}^{s}\left(\eta_{0}\right)$.

Round 1: Each school-age agent of period 1 applies to her most preferred school according to her isolated preferences $\left(\bar{P}_{i}\left(\bar{\eta}_{0}\right)\right.$ in the case of period 1$)$. For each school $s$, let $\bar{p}_{1}^{s 1}$ be the minimum priority score such that the measure of the applicants to $s$ with priority scores $\left(\bar{X}_{i}^{s}\left(\bar{\eta}_{0}\right)\right.$ in the case of period 1) weakly higher than $\bar{p}_{1}^{s 1}$ does not exceed the capacity of school $s, \bar{r}^{s}$. School $s$ rejects all the applicants whose priority score is strictly below $\bar{p}_{1}^{s 1}$ and "holds" the others.

In general, at:

Round $k$ : Each agent who was rejected in the previous round applies to her next choice school according to her isolated preferences $\left(\bar{P}_{i}\left(\bar{\eta}_{0}\right)\right.$ in the case of period 1). Each school $s$ considers the pool of agents that consist of applicants it has been holding and the current applicants. For each school $s$, let $\bar{p}_{1}^{s k}$ be the minimum priority score such that the measure of the agents in the pool of $s$ with priority scores (weakly) higher than $\bar{p}_{1}^{s k}$ does not exceed the capacity of school $s, \bar{r}^{s}$. School $s$ rejects those in the pool whose priority score is strictly below $\bar{p}_{1}^{s k}$ and "holds" the others.

The algorithm terminates when no proposal is rejected and each agent is assigned her final tentative assignment. Let $\overline{\mathbf{p}}_{1}=\left(\lim _{k \rightarrow \infty} \bar{p}_{1}^{s k}\right)_{s \in S}$, which is the period-1 threshold vector associated with the DA-IP.

In period 2, the schools' priority scores are updated based on the period-1 DA-IP matching, $\bar{\eta}_{1}$. In addition, all the school-age agents in this period report their isolated preferences based on the period-1 DA-IP matching, $\bar{\eta}_{1}$. Now using the algorithm

\footnotetext{
${ }^{24}$ The algorithm converges, even though it may require infinite rounds (see Azevedo and Leshno 2016).
} 
described above, we find the period-2 DA-IP matching, $\bar{\eta}_{2}$. Let $\bar{p}_{1}$ be the threshold vector corresponding to period 1 DA-IP matching.

In each period $t \geq 2$, we can run the DA-IP algorithm described above recursively based on the preceding period's DA-IP matching. Let $\overline{\mathbf{p}}_{t}$ be the threshold vector corresponding to period- $t$ DA-IP matching. Also let $\overline{\mathbf{p}}=\left(\overline{\mathbf{p}}_{t}\right)_{t=0}^{\infty}$.

The DA-IP mechanism yields a unique matching in each economy. ${ }^{25}$

\section{A. Strategic Performance of the DA-IP in Continuum Economies}

In this section we compare the strategic performance of the DA-IP under the two priority systems, IPA and Danish. We show that the DA-IP is strategy-proof under IPA, but is manipulable under Danish priorities. The main intuition here is that no student alone can modify, by manipulating her preferences, the threshold scores in the second period. That is, the threshold score vector is immune to single deviations in an environment in which each student has measure zero. ${ }^{26}$ Therefore, if priorities satisfy IPA, an agent that manipulates her preferences will receive a weakly worse allocation in her first period (since the DA is strategy-proof in a static environment) without being able to affect the threshold score in the second period, thus, essentially retaining the same options that she would have without manipulating. We explore this positive result further in the next section for finite large economies. In contrast, if priorities are Danish the DA-IP is manipulable. This happens since agents can affect their own priority score in the second period that they participate.

THEOREM 2 (IPA: Strategy-Proofness of the DA-IP): If the priority system satisfies IPA, then the DA-IP mechanism is strategy-proof in continuum economies.

\section{PROOF:}

Suppose that agent $i$ can manipulate the DA-IP mechanism in some continuum economy $\bar{F}$. At the manipulation, let agent $i$ misreport her preferences as $\succ_{i}^{\prime} \neq \succ_{i}$. Let the economy that results from $i$ 's misreporting be $\bar{F}^{\prime}$. Let $\bar{\eta}$ and $\bar{\eta}^{\prime}$ be the DA-IP matchings in $\bar{F}$ and $\bar{F}^{\prime}$, respectively. Let the threshold scores corresponding to the $\bar{\eta}$ and $\bar{\eta}^{\prime}$ be $\mathbf{p}$ and $\overline{\mathbf{p}}^{\prime}$, respectively. Since the two economies differ in only agent $i$ 's preferences and given that the measure of each agent is 0 , we have that

$$
\overline{\mathbf{p}}=\overline{\mathbf{p}}^{\prime}
$$

Since $i$ can manipulate the DA-IP mechanism at $\bar{F}$, similar to Lemma 1, we obtain that

$$
\left(\bar{\eta}_{t_{i}+1}^{\prime}(i), \bar{\eta}_{t_{i}+1}^{\prime}(i)\right) \succ_{i}\left(\bar{\eta}_{t_{i}+1}(i), \bar{\eta}_{t_{i}+1}(i)\right) \succeq_{i}\left(\bar{\eta}_{t_{i}}(i), \bar{\eta}_{t_{i}}(i)\right) \succeq_{i}\left(\bar{\eta}_{t_{i}}^{\prime}(i), \bar{\eta}_{t_{i}}^{\prime}(i)\right)
$$

\footnotetext{
${ }^{25}$ This result is a straightforward adaptation of the proof by Azevedo and Leshno (2016, theorem 1).

${ }^{26}$ Azevedo and Leshno (2016) notes that small changes in the measure $\eta$ (or in the capacity of each school) can affect the threshold priority score vector significantly. However, note that a zero-measure agent is not able to affect the threshold scores through unilateral deviations.
} 
Set $s \equiv \bar{\eta}_{t_{i}+1}^{\prime}(i)$. The relation above means that $i$ is not matched to $s$ in period $t_{i}$ at both matchings $\bar{\eta}$ and $\bar{\eta}^{\prime}$. Consequently, it must be that $\bar{X}_{i}^{s}\left(\bar{\eta}_{t_{i}}\right)=\bar{X}_{i}^{s}\left(\bar{\eta}_{t_{i}}^{\prime}\right)=x_{i}^{s}$. However, $i$ is matched to $s$ in period $t_{i}+1$ at $\bar{\eta}^{\prime}$ but not at $\bar{\eta}$. In other words, $\bar{X}_{i}^{s}\left(\bar{\eta}_{t_{i}}^{\prime}\right)=x_{i}^{s} \geq \bar{p}_{t_{i}+1}^{s}$ but $\bar{X}_{i}^{s}\left(\bar{\eta}_{t_{i}}\right)=x_{i}^{s}<\bar{p}_{t_{i}+1}^{s}$. However, these two inequalities contradict that $\bar{p}_{t_{i}+1}^{\prime s}=\bar{p}_{t_{i}+1}^{s}$.

In the theorem above, we assumed that the schools' priorities satisfy IPA. For the Danish priority system, the theorem above no longer holds. An agent's preference report can place her at the top of the priority ranking of a school in the subsequent period, provided that she reports this school as her top choice followed by home care. Under the Danish priority system, an agent can change her standing in the schools' priorities of the subsequent period.

THEOREM 3 (Danish Priorities: Manipulation of the DA-IP): If the priority system satisfies Danish priorities, then the DA-IP mechanism is manipulable in some continuum economies.

\section{PROOF:}

Consider an economy in which the threshold score at some school $s$ corresponding to the DA-IP matching is $\bar{p}$ with $\bar{p}_{t}^{s}>0, \bar{p}_{t+1}^{s}>0$ and $t \geq 1$. Consider an agent $i$ who was born in period $t$, and whose preferences satisfy the following two conditions: (i) $(s, s)$ is the most preferred bundle, and (ii) $(h, s)$ $\succ_{i}\left(s^{\prime}, s^{\prime \prime}\right)$ for all $s^{\prime} \neq s$ and $s^{\prime \prime} \neq s$. In addition, suppose that $x_{i}<\min \left\{\bar{p}_{t}^{s}, \bar{p}_{t+1}^{s}\right\}$. Clearly, agent $i$ does not attend $s$ by reporting her preferences truthfully. However, if she reports $s$ as her first choice and $h$ as her second choice, then she will stay home when she is one but attends $s$ when she is two. Thus agent $i$ has a profitable manipulation.

The theorem above implies that there are some economies in which a positive mass of agents may be able to each independently manipulate the DA-IP mechanism for this economy.

\section{Large Markets and Convergence}

Given the positive result of the strategic performance of the DA-IP in continuum economies under IPA, and the important policy implications of this result, in this section we study this strategic performance in large but finite economies. Consider a finite economy $E=(I, \mathbf{r}, X)$. We now define the measure for each finite economy $E$ based on its empirical distribution. Specifically, the measure of each agent $i$ is $\tilde{\nu}(\{i\})=1 /\left|I_{0}\right|$. On the other hand, let the capacities of the schools be $\tilde{\mathbf{r}}=\mathbf{r} /\left|I_{0}\right|$. Using this empirical distribution, we will denote finite economies in a similar fashion to continuum economies. Specifically, let us redefine the finite economies by denoting $\tilde{F}=(\tilde{\nu}, \tilde{\mathbf{r}}, X)$ the finite economy $E$. With this notation we can define the convergence of finite economies to a continuum economy. 
DEFINITION 6: A sequence of finite economies $E^{k}$ converges to a continuum economy $\bar{F}$ if the sequence of economies $\tilde{F}^{k}=\left(\tilde{\nu}^{k}, \tilde{r}^{k}, X\right)$ corresponding to $E^{k}$ satisfies the following two conditions:

(i) $\tilde{\nu}^{k}$ converges to $\bar{\nu}$ in weak ${ }^{*}$ topology;

(ii) $\tilde{r}^{k}$ converges to $\bar{r}$ in supremum norm.

Here observe that if $E^{k}$ converges to $\bar{F}$, then the ratio of the size of agents born in any period $t$ to the size of the agents born in $t-1$ converges to 1 .

In this section, we assume the following assumption in order to ensure the convergence of the stable matchings when the finite economies converge to a continuum economy.

ASSUMPTION 5 (Market Thickness): Consider any continuum economy $\bar{F}$. Then for any $t \geq 0$, any isolated preferences $\bar{P} \in \overline{\mathcal{P}}$ and any $x \ll x^{\prime} \ll \mathbf{1}$,

$$
\bar{\nu}\left(\left\{i: t_{i}=t, \bar{P}_{i}=\bar{P}, \text { and } x \leq x_{i} \leq x^{\prime}\right\}\right)>0 .
$$

The assumption above means that the market is thick in the sense that the distribution of types is continuous. This assumption guarantees the uniqueness of stable matchings in continuum economies. We emphasize that the distribution of types does not have to be the same across periods, so the market might be very different from one period to another.

We now consider what happens to the DA-IP matchings when the sequence of economies converges to a continuum economy. To study this, we first define the distance between two DA-IP matchings (Azevedo and Leshno 2016). Let $\eta$ and $\bar{\eta}$ be the DA-IP matchings of a continuum economy $F$ and of a finite economy $E$. The period $t$ distance between $\bar{\eta}$ and $\eta$ are as follows:

$$
d_{t}(\eta, \bar{\eta})=\left\|p_{t}-\bar{p}_{t}\right\|_{\infty}
$$

Let $d(\eta, \bar{\eta})=\left(d_{t}(\eta, \bar{\eta})\right)_{t=0}^{\infty}$.

Now that the distance between two DA-IP matchings are defined, we can consider the convergence of the DA-IP matchings when the sequence of finite economies converges to a continuum economy.

DEFINITION 7: A sequence of DA-IP matchings, $\left\{\eta^{k}\right\}$, in economies $\left\{E^{k}\right\}$ converges to $\bar{\eta}$ if

$$
\lim _{k \rightarrow \infty} d_{t}\left(\eta^{k}, \bar{\eta}\right)=0 \quad \text { for all } t \geq 1
$$

Now we are ready to present the convergence result of the DA-IP matchings as the sequence of finite economies converges to a continuum economy. 
PROPOSITION 1: If a sequence of finite economies $E^{k}$ converges to a continuum economy $\bar{F}$, then the sequence of DA-IP matchings $\left\{\eta^{k}\right\}$ converges to $\bar{\eta}$.

\section{PROOF:}

See Appendix C.

We are now finally ready to study how the incentives to manipulate the DA-IP mechanism change as the market size grows. For a finite economy $E$, let us define $L_{t}$ as the set of the agents born in period $t$ who benefits by manipulating the DA-IP mechanism in this economy. In the following theorem, we show that as finite economies converge to a continuum economy, the fraction of the agents who can manipulate in finite economies converges to zero.

THEOREM 4: If a sequence of finite economies satisfying IPA, $\left\{E^{k}\right\}$, converges to a continuum economy $\bar{F}$, then $\left\{\left|L_{t}^{k}\right| /\left|I_{t}^{k}\right|\right\} \rightarrow 0$ for each $t \geq 1$.

\section{PROOF:}

See Appendix C.

The main idea of the theorem above is the following: in Lemma 1 we showed that to manipulate the DA-IP mechanism an agent must weakly get worse at her first period, but better in her second period. However, in order to improve her allocation in the second period, she must either lower the threshold scores of the schools, or improve her own score at some school that she was not able to attend in her first period. Due to IPA, the latter is not possible: she can only improve her own score at a school that she attends. Thus, we investigate how likely it is that she might change the threshold score of the schools. As the economy grows, this agent becomes a very small part of the economy, and at some point her manipulation will have a negligible impact on the threshold scores because these are converging to a fixed value, due to Proposition 1. Thus, if an agent can manipulate the DA-IP mechanism in a large economy then her priority score at the school she manages to attain as a result of the manipulation must be very close the threshold score of this school. As the market grows, the proportion of such agents in the economy vanishes. 27

REMARK 1: The market thickness assumption (Assumption 5) can be weakened significantly in Theorem 4. Specifically, once the DA-IP matching is fixed we can define a static continuum economy in each period in which the school age children in that period participate and their preferences are determined by their isolated preferences. ${ }^{28}$ If each such economy has a unique stable matching (in the static sense), then the main result of this paper is still valid. Azevedo and Leshno (2016)

\footnotetext{
${ }^{27}$ Recall that we assumed that in period 0 every agent stays home. This assumption can be relaxed, but one extra minor assumption must be made not to affect our convergence results. As we already mentioned in footnote 12 , we take $\mu_{0}$ and $\bar{\mu}_{0}$ as the primitives of our model. To preserve our main results, we need to assume that the sequence of period-0 matchings in finite economies, $\left\{\mu_{0}^{k}\right\}$, converges to $\bar{\mu}_{0}$ as $E^{k}$ converges to $\bar{F}$.

${ }^{28}$ See Appendix $\mathrm{C}$ for more information.
} 
shows that a wide range of static economies has a unique stable matching. In this sense, the approximate strategy-proofness of the DA-IP matching is valid in a vast array of economies. Theorem 4 is not valid if the limiting continuum economy has multiple stable matchings. We show this point in online Appendix D by presenting an example in which the fraction of agents who can manipulate the DA-IP mechanism does not converge to zero as a sequence of finite economies converge to such a continuum economy.

As a last remark, if the priority system is Danish, then, as in the continuum economies, the fraction of agents who can manipulate the DA-IP does not converge to zero as the market gets large.

\section{Simulation Study}

In the previous section, we showed that the DA-IP becomes approximately strategy-proof as the market size increases. But how large must the market be so that the percentage of agents who can manipulate the DA-IP is small enough? In this section, using randomly generated data we show that a modest market size suffices for this. Therefore, the results in this section complement and reinforce the theoretical results on large markets of the previous section. To conduct this exercise, we first need to figure out whether a given agent has a successful manipulation of the DA-IP. A brute force approach—checking all possible preference reports of an agent-is not feasible, because there are $(|S| !)^{2}$ number of preference reports. ${ }^{29}$ Of course, the brute force approach is not feasible in the static marriage and the many-to-one matching problems either, but in those problems there are two types of strategies - truncating and dropping - that are sufficient to check whether an agent has a successful manipulation of the static DA mechanism. However, as our Example 4 shows, focusing on such strategies could miss some successful manipulations. Therefore, we construct our own algorithm to overcome this complexity problem that we present next.

\section{A. Theoretical Results}

In this section, we present our algorithm that checks if an agent can manipulate the DA-IP.

We first note that for any two-year-old agent the DA-IP mechanism is a static DA mechanism. Thus, given our focus on the domain of preferences satisfying rankability, no two-year old agent has an incentive to misreport her isolated-preference because the DA mechanism is strategy-proof in the static school choice problem. As a result, any manipulating agent must misreport her isolated-preference when she is one year old. Secondly, each manipulating agent's submitted isolated preference report at the period she is age one must alter the period matching of that period. Otherwise, no agent's priority score or isolated

\footnotetext{
${ }^{29}$ In fact, given rankability and the fact that the DA-IP is strategy-proof in a static market, it is enough to check only $|S|$ ! different preference profiles, but this too, very quickly becomes infeasible.
} 
preference differ from the ones under truth-telling. Therefore, we need to find all the DA-IP matchings that follow from a one-year-old agent's isolated preference reports. We will describe how we do this in the next subsection. Before that, let us take this algorithm as given and describe in words how we will proceed to check if there are successful manipulations.

Fix an economy $E$ and an agent $i$. Suppose that agent $i$ is born in period $t \geq 1$.

\section{The Algorithm to Check the Manipulability of the DA-IP Mechanism.}

Step 0: Run the DA-IP mechanism in economy $E$ until period $t+1$ and find the DA-IP matching of $i$. Identify the schools that by attending any of these in period $t$ and her most preferred school in period $t+1, i$ improves over her DA-IP match under truth-telling. Call these schools potentially beneficial.

\section{Step 1:}

(a) Find a period- $t$ matching which is potentially beneficial and can be reached through some isolated-preference misreport of $i$. Denote this matching by $\mu_{t}^{1}$.

(b) Find the isolated preferences, $P\left(\mu_{t}^{1}\right)$, and update the agents priorities, $X\left(\mu_{t}^{1}\right)$. Find the corresponding period $t+1$ matching and call it $\mu_{t+1}^{1}$. If $\left(\mu_{t}^{1}(i), \mu_{t+1}^{1}(i)\right) \succ_{i} \eta(i)$, then stop the algorithm. In this case, $i$ can manipulate the DA-IP mechanism. Otherwise, move to the next step.

\section{Step k:}

(a) Find a period-t matching which is potentially beneficial and has not been considered in the previous steps and can be reached through some isolated-preference report of $i$ in period $t$. Call this matching by $\mu_{t}^{k}$.

(b) Find the isolated preferences, $P\left(\mu_{t}^{k}\right)$, and update the priorities, $X\left(\mu_{t}^{k}\right)$. Find the corresponding period $t+1$ matching and call $\mu_{t+1}^{k}$. If $\left(\mu_{t}^{k}(i), \mu_{t+1}^{k}(i)\right) \succ_{i} \eta(i)$, then stop the algorithm. In this case, $i$ can manipulate the DA-IP mechanism. Otherwise, move to the next step.

If all the period- $t$ matchings to which $i$ can lead by reporting some isolated preference in period $t$ are exhausted, then the DA-IP mechanism is not manipulable. Exhausting all the period- $t$ matchings (part (a) of all the steps) in a feasible amount of time is the most difficult part, and we will describe below the algorithm to achieve this. Finding isolated preferences for a given period in part (b) is straightforward given the preference profile of the agents and the matching in the previous period.

Algorithm to Find All the Matchings to Which an Agent Can Induce.The algorithm that we will present in this section allows us to compute every 
matching that a single agent can induce, given the reports of others. It is possible to improve on this algorithm further (in terms of speed of execution) and the interested readers can find the full version of our algorithm, used in our simulation, in online Appendix D.

This algorithm might be useful in other contexts. For instance, one can compute how a misreport (by simple error or a calculated strategic manipulation) by a single agent can affect the entire matching. Also one perhaps can study different properties of the DA mechanism. For example, it is well-known that the DA mechanism is bossy ${ }^{30}$, i.e., an agent sometimes can misreport her preference and alter others' allocation without changing her own allocation. With the help of our algorithm one can figure out how often an agent has a bossy manipulation.

In this section, we focus on static economies. We refer to economies defined in Section I as dynamic economies. The notations $S=\left\{h, s_{1}, \ldots, s_{m}\right\}$ and $\mathbf{r}=\left(r^{s}\right)_{s \in S}$ indicate the set of schools and their capacities as before. The same assumptions, $r^{s}<\infty$ for all $s \neq h$ and $r^{h}=\infty$, are satisfied here too. However, each agent $j$ in static economies is characterized by a pair $\left(P_{j}, x_{j}\right)$, where $P_{j}$ is $j$ 's strict preferences over $S$ (not over the set of possible pairs of schools) and $x_{j}=\left(x_{j}^{S}\right)_{s \in S} \in[0,1]^{m+1}$ is $j$ 's priority scores at schools. Let $J$ be the set of agents and a pair $(J, r)$ is a static economy. Here, every agent wants to get matched to only one school and we use the DA mechanism for each static economy. A typical matching in static economy $(J, r)$ is denoted by $\sigma$.

Fix $i \in J$, and we now find all the DA matchings to which $i$ 's preference reports in economy $(J, r)$ can lead. In other words, we vary $i$ 's preference report while fixing everyone else's preferences as in economy $(J, r)$. To simplify our presentation, we say a matching $\sigma$ is attainable if there exists a preference report $P_{i}^{\prime}$ such that the DA mechanism produces $\sigma$ if $i$ submits $P_{i}^{\prime}$ (while the other agents submit their preference reports truthfully).

Here the number of $i$ 's preference reports in a static economy explodes very quickly with the number of schools. For instance, in a world with 10 schools and the possibility of staying home, an agent $i$ has 11 ! (or more than 39 million) preference reports. Therefore, finding the attainable matchings using brute force is not feasible. Fortunately, there is an easier way to achieve our goal, but we first present a concrete example that is repeatedly used in this section.

\footnotetext{
${ }^{30}$ Dubins and Freedman (1981) show that the DA is strategy-proof but not group strategy-proof. Pápai (2000) demonstrates that the group strategy-proofness is equivalent to strategy-proofness and nonbossiness. Based on these, the DA is bossy. See also Kojima (2010).
} 
Example 5: There are 11 schools (including $h$ ) and 10 agents. Each school has a capacity of one agent per period. Below we present the preferences and priorities of the schools: ${ }^{31}$

$\begin{array}{rlllllllllll}i_{1}: & s_{1}, & s_{3}, & s_{9}, & s_{10}, & s_{8}, & s_{4}, & s_{2}, & s_{6}, & s_{7}, & s_{5}, & h \\ i_{2}: & s_{4}, & s_{8}, & s_{1}, & s_{10}, & s_{2}, & s_{9}, & s_{3}, & s_{5}, & s_{7}, & s_{6}, & h \\ i_{3}: & s_{2}, & s_{6}, & s_{8}, & s_{10}, & s_{4}, & s_{7}, & s_{3}, & s_{9}, & s_{5}, & s_{1}, & h \\ i_{4}: & s_{3}, & s_{9}, & s_{4}, & s_{2}, & s_{5}, & s_{1}, & s_{6}, & s_{10}, & s_{8}, & s_{7}, & h \\ i_{5}: & s_{9}, & s_{8}, & s_{10}, & s_{4}, & s_{5}, & s_{6}, & s_{3}, & s_{7}, & s_{2}, & s_{1}, & h \\ i_{6}: & s_{4}, & s_{2}, & s_{1}, & s_{6}, & s_{10}, & s_{8}, & s_{9}, & s_{3}, & s_{5}, & s_{7}, & h \\ i_{7}: & s_{3}, & s_{8}, & s_{2}, & s_{10}, & s_{6}, & s_{1}, & s_{4}, & s_{9}, & s_{7}, & s_{5}, & h \\ i_{8}: & s_{2}, & s_{8}, & s_{3}, & s_{10}, & s_{1}, & s_{5}, & s_{6}, & s_{4}, & s_{7}, & s_{9}, & h \\ i_{9}: & s_{9}, & s_{10}, & s_{6}, & s_{2}, & s_{7}, & s_{4}, & s_{1}, & s_{8}, & s_{5}, & s_{3}, & h \\ i_{10}: & s_{1}, & s_{6}, & s_{8}, & s_{10}, & s_{3}, & s_{4}, & s_{2}, & s_{9}, & s_{7}, & s_{5}, & h\end{array}$

$\begin{array}{rcccccccccc}s_{1}: & i_{2}, & i_{9}, & i_{1}, & i_{10}, & i_{8}, & i_{5}, & i_{4}, & i_{7}, & i_{6}, & i_{3} \\ s_{2}: & i_{2}, & i_{6}, & i_{10}, & i_{8}, & i_{9}, & i_{4}, & i_{1}, & i_{7}, & i_{3}, & i_{5} \\ s_{3}: & i_{1}, & i_{7}, & i_{3}, & i_{9}, & i_{2}, & i_{5}, & i_{4}, & i_{8}, & i_{6}, & i_{10} \\ s_{4}: & i_{10}, & i_{9}, & i_{1}, & i_{7}, & i_{4}, & i_{3}, & i_{5}, & i_{2}, & i_{6}, & i_{8} \\ s_{5}: & i_{6}, & i_{8}, & i_{9}, & i_{5}, & i_{4}, & i_{10}, & i_{1}, & i_{2}, & i_{3}, & i_{7} \\ s_{6}: & i_{8}, & i_{7}, & i_{3}, & i_{2}, & i_{10}, & i_{4}, & i_{9}, & i_{6}, & i_{1}, & i_{5} \\ s_{7}: & i_{5}, & i_{3}, & i_{10}, & i_{2}, & i_{4}, & i_{6}, & i_{8}, & i_{7}, & i_{1}, & i_{9} \\ s_{8}: & i_{4}, & i_{10}, & i_{9}, & i_{1}, & i_{5}, & i_{3}, & i_{2}, & i_{8}, & i_{6}, & i_{7} \\ s_{9}: & i_{8}, & i_{1}, & i_{7}, & i_{6}, & i_{4}, & i_{2}, & i_{5}, & i_{9}, & i_{3}, & i_{10} \\ s_{10}: & i_{1}, & i_{8}, & i_{9}, & i_{4}, & i_{10}, & i_{7}, & i_{6}, & i_{5}, & i_{3}, & i_{2}\end{array}$

There are $11 !\left(\cong 3.9 \times 10^{7}\right)$ possible preference reports that agent $i_{1}$ can submit, but it turns out that these reports lead to only 10 different matchings.

We will need several new notions to present our algorithm to find all the attainable matchings. If $i$ is matched to $s$ under some attainable matching then we say a school $s$ is attainable (for agent $i$ ). Otherwise, $s$ is non-attainable. We reserve the notations $S^{A}$ and $S^{N A}$ for the set of attainable and non-attainable schools, respectively. We note that $h$ is attainable because agent $i$ is assigned to $h$ if she ever applies to $h$ at any round of the DA algorithm. A simple way to check if a given school $s$ is attainable is to find the DA matching when $i$ places $s$ at the top of her preference report. However, one needs to run the DA algorithm $|S|$ times. This number can be decreased significantly due to the lemma below (the proof is in online Appendix D). 
LEMMA 2: Let $s$ be an attainable school. The DA allocates $i$ to $s$ if and only if $i$ submits a preference report which lists only non-attainable schools ahead of s.

The lemma above is a direct consequence of the fact the DA mechanism is strategy-proof in static settings. It says that the DA mechanism matches agent $i$ to the highest ranked attainable school in her preference report. This allows us to construct a simple algorithm to find all the attainable schools. First, $i$ modifies her true preferences so that $h$ is the least preferred school. We then find the DA match of $i$ corresponding to this report. By Lemma 2, we have identified the first attainable school. To find the second one we run the DA algorithm when $i$ modifies the previous report only in that the first attainable school is the least preferred. The modified report is identical to the original one up till the first attainable school appears in the original report. Consequently, the DA algorithm produces the identical result until the round in which $i$ applies to different schools under the two reports. This suggests that after finding the first attainable school, one should reset the DA algorithm to the round in which $i$ applied to this school. Now we continue with the DA algorithm but by making $i$ apply to the school that follows the first attainable in her preference report. By repeating this process until $i$ matches to $h$, one finds all the attainable schools.

The Algorithm to Find the Set of Attainable and Non-Attainable SchoolsSet $S^{A}=\emptyset$.

Step 1: Agent $i$ updates her true preference report by placing $h$ at the very end of her list. Everyone else reports one's own true preferences. This step consists of several rounds. Set the reset round for step 1 , denoted by $R R^{1}$, to 1 . Go through the same rounds as in the standard DA algorithm, but if $i$ is rejected in some round $k$, then set $R R^{1}$ to $k+1$. Step 1 concludes at the round in which no one is rejected. Assign the agents to the schools that hold them in this round and call this matching $\sigma^{1}$. Add $\sigma^{1}(i)$ to $S^{A}$. If $\sigma^{1}(i)=h$, then terminate the algorithm. By construction, agent $i$ applied to $\sigma^{1}(i)$ at round $R R^{1}$ and was last rejected by some school at round $R R^{1}-1$.

Step $\kappa$ : Update agent $i$ 's preference report of Step $\kappa-1$ by placing $s^{\kappa-1}$ to the very end. This step starts from the reset round found in the previous step. That is, we assume that the application and rejection rounds until the reset round of the previous step have already happened in the DA algorithm. Set the reset round of this step, $R R^{\kappa}$, to $R R^{\kappa-1}$. As in Step 1, proceed as in any standard round of the DA algorithm (while keeping the track of the reset round) until the round in which no agent is rejected. Assign the agents to the schools which hold them and call this matching $\sigma^{\kappa}$. Add $\sigma^{\kappa}(i)$ to $S^{A}$. Terminate the algorithm if $\sigma^{\kappa}(i)=h$. Otherwise, proceed to the next step.

The algorithm must terminate because at each step, the schools that have rejected $i$ as well as the unacceptable schools for $i$ grow. Thus, at some step of the algorithm $i$ applies to $h$, and is matched to it. The set of attainable schools is $S^{A}$ found at the last step of the algorithm. 
Due to Lemma 2, the algorithm finds the highest ranked attainable school in $i$ 's report of a given step. In addition, $i$ 's report in any step ranks the attainable school found in the preceding step after $h$. As a result, the algorithm not only yields a new attainable school at each round, but also finds all the attainable schools in the exact order that were listed in i's very first report. Consequently, the number of steps at which the algorithm stops is the number of attainable schools. ${ }^{32}$

In Example 5, there are seven attainable and four non-attainable schools for agent 1, i.e., $S^{A}=\left\{s_{3}, s_{4}, s_{5}, s_{7}, s_{9}, s_{10}, h\right\}$ and $S^{N A}=\left\{s_{1}, s_{2}, s_{6}, s_{8}\right\}$.

We say a matching $\sigma$ is $s$-attainable if (i) $\sigma$ is attainable and (ii) $i$ is matched to $s$. For some attainable school $s$, let us find all the $s$-attainable matchings. Lemma 2 shows that if $i$ is to get allocated to $s$, then she has to rank $s$ as the highest ranked attainable school in her report. In other words, the schools $i$ ranks ahead of $s$ must all be non-attainable. A useful result is that the DA produces the same matching for two reports of $i$ that rank the same non-attainable schools ahead of $s .{ }^{33}$ Therefore, the maximal number of reports needed for finding the $s$-attainable matchings is $2^{\left|S^{N A}\right|}$ - the number of all subsets of $S^{N A}$. Thus, in Example 5, the original 39 million plus strategies reduce to no more than $16 \times 7$ to find all the attainable matches.

The number $2^{\left|S^{N A}\right|}$ is still unmanageable if there are many non-attainable schools. Fortunately, it turns out that depending on the attainable school, we can dispose of some non-attainable schools. Specifically, once $i$ fixes the relative positioning of the remaining schools in her report where she places such schools does not affect the resulting DA matching. Thus, for each attainable school $s$, we now split the non-attainable schools into two groups: redundant and non-redundant.

DEFINITION 8: Fix $s \in S^{A}$. A school s' is s-redundant if the DA produces the same matching when $i$ submits any two preference reports, $P_{i}^{s}$ and $\tilde{P}_{i}^{s}$, where:

(i) in both reports $P_{i}^{s}$ and $\tilde{P}_{i}^{s}$, s is the highest ranked attainable school; and

(ii) the sets of schools ranked higher than $s$ under $P_{i}^{s}$ and $\tilde{P}_{i}^{s}$ differ only in that the one under $P_{i}^{s}$ does not contain $s^{\prime}$ while the one under $\tilde{P}_{i}^{s}$ does.

A non-attainable school $s^{\prime}$ is s-non-redundant if $s^{\prime}$ is not redundant for $s$. We use the notations $S^{R}(s)$ and $S^{N R}(s)$ to denote the s-redundant and s-non-redundant schools, respectively.

\footnotetext{
${ }^{32}$ For the purpose of finding if the DA-IP is manipulable or not by $i$, all the schools that are not potentially beneficial should be dropped from $S^{A}$

${ }^{33}$ Note that each DA matching corresponding to one of these preferences of $i$ is stable in a static sense in the economy corresponding to the other report of $i$. Also, recall that each DA matching in any static economy is the agent optimal stable matching in that economy. Combining this with the fact that agent $i$ is matched to school $s$ at both DA matchings, we find that each DA matching weakly Pareto dominates the other. This of course means that the two DA matchings must be the same.
} 
Where $s$-redundant schools are ranked in $i$ 's report does not lead to additional DA matchings as long as $s$ is the highest ranked attainable school. Thus, by identifying the redundant schools one can significantly decrease the number of preference reports leading to different DA matchings. But how can we identify these schools? Below we present an alternative characterization of redundant schools which is easy to work with. Let us first fix the required notation. Fix an attainable school $s$ and write $\hat{P}_{i}^{s}$ to denote a preference report of $i$ in which $s$ is the top ranked school. We write $\hat{\sigma}^{s}$ to denote the DA matching when $i$ reports $\hat{P}_{i}^{s}$.

LEMMA 3: Fix $s \in S^{A}$. A school $s^{\prime}$ is s-redundant if and only if the priority score of agent $i$ at school $s^{\prime}$ is lower than the priority score of those who are matched to $s^{\prime}$ under $\hat{\sigma}_{t}$, i.e.,

$$
x_{i}^{s^{\prime}}<\min _{j \in \hat{\sigma}\left(s^{\prime}\right)}\left\{x_{j}^{s^{\prime}}\right\}
$$

\section{PROOF:}

See the proof of Lemma 9 in the online Appendix D.

The intuition of the lemma above as follows: Consider $\hat{P}_{i}^{s}$, which by construction ranks $s$ as the most preferred. If $s^{\prime}$ is $s$-redundant, then ranking $s^{\prime}$ ahead of $s$ does not alter the DA matching by definition. Because $i$ matches to $s, i$ must have been rejected from $s^{\prime}$ at some point in the DA algorithm. This means that in order for $i$ to be rejected from $s^{\prime}$, those who are matched to $s^{\prime}$ must have better priorities than $i$ at $s^{\prime}$. This is the exact condition needed for redundancy.

Lemma 3 gives us an easy way to find the non-redundant schools for each attainable school. That is, one first needs to find the DA matching when $i$ ranks a given attainable school $s$ first in her preference report. If some agent who is matched to an unattainable school $s^{\prime}$ has a lower priority than $i$ in that school then $s^{\prime}$ is $s$-non-redundant. Consequently, to find the set of $s$-attainable matchings, one needs to find all the subsets of non-redundant schools for $s$ and place each subset ahead of $s$ in $i$ 's report and run the DA algorithm for each report. This process yields all the $s$-attainable matchings. Here, one only considers $2^{\left|S^{N R}(s)\right|}$ possible preference reports.

Let us now revisit Example 5 in which $S^{A}=\left\{s_{3}, s_{4}, s_{5}, s_{7}, s_{9}, s_{10}, h\right\}$ and $S^{N A}=\left\{s_{1}, s_{2}, s_{6}, s_{8}\right\}$ for agent 1 . Let us find the $h$-non-redundant schools. When agent 1 ranks $h$ first in her report then the following DA match emerges:

$$
\begin{aligned}
& s_{1} \leftrightarrow i_{10} \quad s_{2} \leftrightarrow i_{6} \quad s_{3} \leftrightarrow i_{7} \quad s_{4} \leftrightarrow i_{2} \quad s_{6} \leftrightarrow i_{3} \\
& s_{7} \leftrightarrow i_{9} \quad s_{8} \leftrightarrow i_{5} \quad s_{9} \leftrightarrow i_{4} \quad s_{10} \leftrightarrow i_{8} \quad h \leftrightarrow i_{1}
\end{aligned}
$$

At schools $s_{1}$ and $s_{8}$, agent $i_{1}$ has a higher priority than the respective matches of these schools, i.e., $i_{10}$ and $i_{5}$. The same cannot be said for $s_{2}$ and $s_{6}$. Thus, $s_{1}$ and $s_{8}$ are $h$-non-redundant, while $s_{2}$ and $s_{6}$ are $h$-redundant. By following the same steps, we find that only two other attainable schools, namely $s_{5}$ and $s_{10}$, have non-redundant schools. In both cases, $s_{1}$ and $s_{8}$ are non-redundant.

We next present two more results that speed the process of finding attainable matchings. The first one says that the set of $h$-non-redundant schools is larger than 
the one for any other attainable school. Consequently, if there is no $h$-non-redundant school then there is only one attainable matching per each attainable school. Recall that we identify one attainable matching in each step of the algorithm to find all the attainable schools. Hence, in some cases, the process of finding the attainable schools can identify all the attainable matchings.

LEMMA 4: Fix an attainable school $s \neq h$. If school $s^{\prime}$ is $s$-non-redundant, then $s^{\prime}$ is h-non-redundant.

\section{PROOF:}

See online Appendix D.

Ideally, if two reports of $i$ lead to the same DA matching, then only one of them should be considered. The next lemma helps in this direction. Fix two different reports produce the same DA matching which matches $i$ to $s$. Then these two reports continue to produce the identical matchings even if they include more non-attainable schools ahead of $s$.

LEMMA 5: Fix $s \in S^{A}, S \subset S^{N A}$, and $\tilde{S} \subset S^{N A}$. If two preference reports of agent $i$ respectively placing the members of $S$ and $\tilde{S}$ ahead of $s$ lead to the same $D A$ matching then for any $S^{\prime} \subset S^{N A}$, any two reports of $i$ placing $S^{\prime} \cup S$ and $S^{\prime} \cup \tilde{S}$ respectively ahead of s lead to the same DA matching.

\section{PROOF:}

See online Appendix D.

To find all the attainable matchings, we run the DA algorithm for various preference reports of agent $i$. Because all the other agents report their true preferences, the DA goes through many similar application and rejection cycles. To mitigate this problem, we use the result of McVite and Wilson (1971) that the DA algorithm can be run sequentially. To be precise, in each round of their version of the DA, only one agent (who was either rejected in the previous round or has not made any applications yet) applies to the highest ranked school that has not rejected her previously, and the school rejects the lowest ranked agent among those whom it has been holding plus the new applicant if there are no free spots. In this algorithm, let $i$ be the last agent to apply to any school. Then the tentative matching right before $i$ makes her first proposal is the DA matching corresponding to $i$ 's report which places $h$ at the top of $i$ 's report. We call this matching by $\sigma^{*}$. To find the DA matching corresponding to any report of $i$, we set the tentative matching right before $i$ 's first application to be $\sigma^{*}$ and continue with the McVittie and Wilson version of the DA from the round in which $i$ applies to a school the first time. The time we save this way when the DA is repeatedly run is significant.

The Algorithm to Find the Attainable Matchings. - This algorithm considers all the attainable schools sequentially in each step starting with $h$. Each step could consist of several rounds. Order the members of $S^{A}$ so that $\left\{h, s^{1}, \ldots, s^{\left|S^{A}\right|-1}\right\}$. 
Step 0: Fix a preference report of $i$ in which $h$ is ranked first. Compute the DA matching, denoted by $\sigma^{0}$, under this report and using Lemma 3, find all the $h$-non-redundant schools, i.e., $S^{N R}(h)$. If $S^{N R}(h)=\varnothing$, then consider the algorithm to find the attainable schools. Recall that in each step of that algorithm, one attainable school and matching is identified. These are the only attainable matchings and terminate the algorithm. If $S^{N R}(h) \neq \emptyset$, then let $S^{N R}(s)=\left\{S: S \subseteq S^{N R}(s)\right\}$. Order the elements in this set $S^{N R}(h)$ in increasing number of elements that each of the subsets contains. For example, the first element in the set will have only one school. When two sets have the same number of elements, rank them randomly. We now find the set of $h$-attainable matchings denoted by $\Sigma(h)$. Initially, $\Sigma(h)=\left\{\sigma^{0}\right\}$.

Round 1. Consider the first element $S^{N R}(h)$. Let $i$ place the single school in this element ahead $h$ in her report. Find the DA matching corresponding to this report and add it to $\Sigma(h)$.

Round $k$. Consider the $k$ th element of $S^{N R}(h)$ denoted by $S^{k}$. Let $i$ place only the schools in $S^{k}$ ahead of $h$ in her report. Find the DA matching corresponding to this report and if it is different than the $h$-attainable matchings identified in the previous rounds, then add to $\Sigma(h)$. If not, delete all the elements of $S^{N R}(h)$ that contain $S^{k}$ and comes after $S^{k}$. Step 0 ends once we have considered all the elements of $S^{N R}(h)$ and we continue to the next step.

Step $\kappa$ : Fix a preference report of $i$ in which $s^{\kappa}$ is ranked first. Compute the DA matching, denoted by $\sigma^{\kappa}$ under this report and using Lemma 3, find all the $s^{\kappa}$-non-redundant schools, i.e., $S^{N R}\left(s^{\kappa}\right)$. Initially, the set of $s^{\kappa}$-attainable matchings, $\Sigma\left(s^{\kappa}\right)=\left\{\sigma^{\kappa}\right\}$. If $S^{N R}\left(s^{\kappa}\right)=\emptyset$, then we are done with this step. If $S^{N R}\left(s^{\kappa}\right) \neq \varnothing$ then find all the $s^{\kappa}$-attainable matchings by using the same algorithm used in Step 1.

The algorithm ends once we have exhausted all the elements of $S^{A}$ and the set of all attainable matchings is $\Sigma(h) \cup \Sigma\left(s^{1}\right) \cup \cdots \cup \Sigma\left(s^{S^{A} \mid-1}\right)$.

PROPOSITION 2: The algorithm above yields all the matchings that are the result of the DA mechanism for some report of $i$ and in which $i$ is allocated to s.

\section{PROOF:}

This proposition is a simple consequence of Lemmas 3, 4, and 5.

To find all the $h$-attainable matchings in our Example 5, we consider only fourdifferent preference reports that place different subsets of $h$-non-redundant schools for $h$, i.e., $\left\{s_{1}, s_{8}\right\}$, ahead of $h$ in agent 1 's report. It turns out that placing only $s_{1}$ or $s_{8}$ ahead of $h$ lead to the same DA matching. Thus, we do not need to consider the case in which both $s_{1}$ and $s_{8}$ are placed ahead of $h$. The four of them leads to only three different DA matchings under which, of course, agent 1 is matched to $h$.

Our algorithm significantly reduces the complexity if compared to the brute force approach. The number of reports used in our algorithm depends on the example. For instance, in Example 5, the brute force algorithm would consider 
$11 ! \approx 39$ million preference reports and run the same number of DA algorithms. On the other hand, our algorithm only runs the DA 20 times: 7 to find attainable schools, 7 to find redundant schools ( 1 for each attainable school) and 6 more to find the DA matchings under which agent 1 is matched to $s_{5}, s_{10}$, or $h$.

\section{B. Simulation Results}

In this section, we generate markets randomly and then estimate the percentage of the markets in which a given agent has a successful manipulation of the DA-IP mechanism. Given that each agent participates in the assignment system twice, we only consider a two-period version of our model. Specifically, one-third of the agents are two years old and another one-third are one year old in period 1. The remaining one-third of the agents are one year old in period 2. As in the model, in each period only school age children participate in the DA-IP mechanism of that period. We vary the number of schools, the schools' capacities, and the number of the agents. However, we assume that each market is balanced, i.e., the number of agents that the schools can admit in each period is the same as the number of the school-age agents in that period.

We will consider different ways of generating the primitives of the preferences in the economy. However, in all versions considered, each agent's payoff from attending two schools in two periods is the sum of the payoffs the agent obtains by holding a spot at these schools, i.e., the agents' preferences are strongly rankable. The agents' priority scores are drawn according to a uniform distribution on the $[0,1]$ interval. We consider both Danish priority and the one satisfying IPA.

In each simulation exercise we vary the number of schools and the schools' capacities. For each combination, we randomly generate 10,000 markets, and in each market we check whether agent \#1 can manipulate the DA-IP mechanism using the algorithm we proposed in the previous section.

We first consider the cases in which the agents have correlated preferences. We model this by assuming that there is an underlying ranking of schools which affects the agents' payoffs. To be specific, the payoff of each agent $i$ from a school consist of (i) a deterministic part which is common to everyone and (ii) a random part which is individual specific and varies with schools. Formally,

$$
\begin{aligned}
u_{i}\left(s_{k}\right) & =\frac{m+1-k}{m}+\epsilon_{i}\left(s_{k}\right) \quad \forall s_{k} \in\left\{s_{1}, \ldots, s_{m}\right\} \quad \text { and } \\
u_{i}(h) & =\epsilon_{i}(h),
\end{aligned}
$$

where $\epsilon_{i}(s)$ for $s \in\left\{h, s_{1}, \ldots, s_{m}\right\}$ is randomly drawn according to the uniform distribution on the $[0,1]$ interval. In addition, the random shocks are independent of each other. Observe here that in expectation, homecare yields the worst payoff and the lower indexed schools result in better payoffs than their higher indexed counterparts. In terms of expected payoffs, two schools with consecutive indices differ by $1 / m$. For the same two schools, the probability that the lower indexed one yields a higher payoff is $0.5+(2 m-1) / 2 m^{2}$, which translates to approximately 
Table 1-(Correlated Preferences): The Percentage of Markets in Which an Agent Can Manipulate the DA-IP Mechanism

\begin{tabular}{lccccccc}
\hline \hline \multirow{2}{*}{$\begin{array}{c}\text { Schools' } \\
\text { capacity }\end{array}$} & \multicolumn{3}{c}{ Priorities satisfying IPA } & & \multicolumn{3}{c}{ Danish priorities } \\
\cline { 2 - 4 } \cline { 6 - 8 } \cline { 6 - 8 } & 10 schools & 50 schools & 100 schools & & 10 schools & 50 schools & 100 schools \\
\hline 1 & $0.97 \%$ & $0.98 \%$ & $0.99 \%$ & & $19.3 \%$ & $28.75 \%$ & $31.42 \%$ \\
10 & $0.29 \%$ & $0.36 \%$ & $0.30 \%$ & & $26.42 \%$ & $29.37 \%$ & $35.90 \%$ \\
15 & $0.20 \%$ & $0.11 \%$ & $0.13 \%$ & & $26.83 \%$ & $34.90 \%$ & $36.83 \%$ \\
20 & $0.14 \%$ & $0.08 \%$ & $0.10 \%$ & & $27.04 \%$ & $34.96 \%$ & $37.73 \%$ \\
& $0.06 \%$ & $0.07 \%$ & $0.08 \%$ & & $28.11 \%$ & $35.79 \%$ & $36.51 \%$ \\
\hline
\end{tabular}

0.51 in the case of 100 schools. We report our results in Table 1, and for each case we generated 10,000 markets.

Under priorities satisfying IPA, the percentage of markets in which a given agent can manipulate DA-IP mechanism is low in general, and this number drops sufficiently close to zero as the schools' capacities increase when the priorities satisfy IPA. For instance, if the schools' capacities are 20 , then agent \#1 can manipulate in 8 out of the 10,000 markets with 100 schools. The capacity of 20 agents is small if we consider the typical day care institutions.

When the priorities are Danish, the manipulation percentages are strikingly higher than those under priorities satisfying IPA. Clearly, the agents have now an extra channel of manipulation which is to stay home in period 1. By staying home in period 1, one improves her priority scores near the top in the second period for each school. Consequently, the manipulating agent would almost surely attain her favorite school in the second period, thus gaining in terms of the payoff in that period. However, staying home or any other manipulation is costly in period 1 because this period's allocation is worse. Here, with correlated preferences, the numbers for manipulation under Danish priorities are the highest ones in our exercises: the percentage of the manipulable markets ranges from 19 percent to 38 percent depending on the number of schools and their capacities.

We now consider the case in which the agents' payoffs from holding a spot at a school (including homecare) are drawn according to a uniform distribution on the $[0,1]$ interval. ${ }^{34}$ In other words, there is no underlying ranking of the schools. Each payoff for each agent is drawn independently. In the previous exercise, with correlated preferences, the lower indexed schools were highly demanded. Thus, these schools were able to pick among their most preferred applicants. In this sense, the situation is comparable to the cases in which the school-proposing DA is used. In the new simulation exercise below, the agent proposing DA is much more favorable to the agents. In Table 2 we present the percentage of markets in which agent \#1 has a successful manipulation.

Here, when the priorities satisfy IPA, the percentage of markets in which a given agent can manipulate DA-IP mechanism is again low in general, and this number

\footnotetext{
${ }^{34}$ We also considered cases in which the payoff from homecare is zero. In other words, the cost of staying home is very high. The manipulation percentages are higher than the ones we obtain here and reach 3.62 percent in some cases when the capacity is 1 . However, they become negligible as the capacities increase. We include these results in the online Appendix.
} 
Table 2-The Percentage of Markets in Which a Given Agent Can Manipulate THE DA-IP MECHANISM

\begin{tabular}{lccccccc}
\hline \hline \multirow{2}{*}{$\begin{array}{c}\text { Schools' } \\
\text { capacity }\end{array}$} & \multicolumn{3}{c}{ Priorities satisfying IPA } & & \multicolumn{3}{c}{ Danish priorities } \\
\cline { 2 - 4 } \cline { 6 - 7 } \cline { 6 - 7 } & 10 schools & 50 schools & 100 schools & & 10 schools & 50 schools & 100 schools \\
\hline 1 & $0.58 \%$ & $1.48 \%$ & $1.67 \%$ & & $2.87 \%$ & $2.57 \%$ & $2.74 \%$ \\
10 & $0.20 \%$ & $0.47 \%$ & $0.60 \%$ & & $1.63 \%$ & $1.26 \%$ & $1.07 \%$ \\
15 & $0.05 \%$ & $0.23 \%$ & $0.25 \%$ & & $1.05 \%$ & $0.94 \%$ & $0.77 \%$ \\
20 & $0.03 \%$ & $0.15 \%$ & $0.22 \%$ & & $0.75 \%$ & $0.77 \%$ & $0.41 \%$ \\
& $0.02 \%$ & $0.15 \%$ & $0.17 \%$ & & $0.67 \%$ & $0.53 \%$ & $0.39 \%$ \\
\hline
\end{tabular}

drops sufficiently close to zero as the schools' capacities increase. For instance, if the schools' capacities are 20 , then agent \#1 can manipulate in 17 out of the 10,000 markets with 100 schools. Again the trend that the manipulation percentage drops with the schools' capacity remains the same.

When the priorities are Danish, the manipulation percentages are higher than those under priorities satisfying IPA but the magnitude drops significantly compared to the ones under correlated preferences. Here, there is no differential competition for the schools in both periods while before there was very fierce competition for lower indexed schools. Consequently, under uncorrelated preferences, the potential benefit of staying home becomes relatively low. Competition for the schools is the main driving force for manipulation being high under Danish priorities. Indeed, if we introduce competition in markets with uncorrelated preferences (by considering unbalanced markets and increasing the number of students), the manipulation rates under Danish priorities increases significantly. 35

Finally, we emphasize that in all of our simulations we assumed that an agent who is contemplating to misreport her preference knows everyone else's preferences. This, of course, is a very strong assumption in reality. To analyze this question in a bit more detail, we study how one's manipulation performs if there is incomplete information. Specifically, we assume that an agent who is about to misreport her preference knows the payoffs and priorities of the agents who are born at the same time period as her, but not of those who are born in the subsequent period. We first fix such an agent and consider markets with 50 schools that have one spot each. Then, we generate 5,000 markets using the same method in our first simulation exercise where the agents have correlated preferences. We focus on the markets in which agent 1 has a successful manipulation. To introduce incomplete information, for each of these markets we generate 1,000 new variations of them. In each variation, we fix the payoffs and priorities for the agents born in period 1 while randomly generating new payoffs and priorities for the agents who arrive in the second period. Thus, we can now calculate the expected payoff of the agent under truth telling and

\footnotetext{
${ }^{35}$ With correlated preferences, competition for the best schools leads to higher manipulation rates. It is important to highlight that in unbalanced markets there are two opposing forces. Competition means that agents are being allocated to lower ranked schools on average, which suggests that manipulation is more likely to be successful. On the other hand, competition in unbalanced markets also forces many students out of school under truth-telling. These students will not be able to manipulate under Danish prioritities. In addition, those who manipulate by staying home do not get better priority than those who are forced to stay home. This again reduces the possibility of manipulation under Danish prioirities. We include some simulation results for unbalanced markets in the online Appendix.
} 
any other strategy. To avoid the computational complexity, we focus on the very first reports that were found as successful manipulations in the original markets instead of all the possible preference reports.

Under priorities satisfying IPA, there were 52 markets in which agent 1 can manipulate. The expected payoff of the agent from the manipulation exceeded the one from truth telling in 2 cases only when incomplete information is introduced. On average, the payoff from truth telling was 8.5 percent higher. Furthermore, out of all the possible 52,000 markets $(52 \times 1,000)$, manipulation was a better strategy in 2,744 cases while truth telling was better in 44,369 cases. This means that any manipulation of the DA-IP in one specific market is not likely to succeed in other markets if the payoffs for the agents who arrive later are randomly drawn. Although our assumption on the payoff distribution is somewhat specific, we believe that this exercise reinforces our general conclusion that the manipulation of the DA-IP in dynamic markets is not a significant problem.

When priorities are Danish, we found that there were 1,439 cases in which an agent can manipulate. Out of these, staying home was the manipulation strategy in 1,398 cases which led to $1,398,000$ newly generated markets. Staying home outperformed truth telling in 90.6 percent of the these markets with an average margin of 12.8 percent. In terms expected payoffs, in 92 percent of the 1,398 markets, staying home was superior to truth telling.

\section{Conclusion}

In this paper we have studied the strategic incentives in the DA mechanism in dynamic matching markets. The DA mechanism is manipulable in a dynamic school choice model, and here we analyzed how manipulable it is in a dynamic large market. We showed that under a suitable restriction on the schools' priorities, there is a large market relief. Specifically, we proved that if each school's priority is affected by the previous period's matching only through previously enrolled agents, then the period-by-period DA mechanism is approximately strategy-proof when the schools' capacities as well as the number of participating agents is large. We also show that without this restriction the mechanism might remain manipulable even in large markets, as we illustrate with the case of the priority system in Danish day cares. An interesting question here is whether incomplete information would make agents less likely to manipulate.

Further, we constructed an algorithm that checks each possible deviation for the agent in a dynamic market. The fact that the DA fails non-bossiness, implies that there is a significant computational complexity in checking each possible deviation. Thus, we constructed our own algorithm to overcome this complexity. Our construction relies on well-known properties of the DA and we focus on the attainable matchings by a single agent, matchings that an agent can unilaterally induce fixing others' reports. We believe that this algorithm might be of independent interest. For example, one might investigate how manipulable is a mechanism that improves efficiency by combining DA with TTC. Another interesting question is to investigate the impact of a single agent in the entire matching. If an agent misreports her action by mistake, for example, what is the average impact of 
this mistake in the entire economy. Our algorithm provides a tool to answer these questions.

With our algorithm, we have done simulations based on randomly generated data to have a better assessment of how large a market must be in order for us to consider that the market is almost strategy-proof assuming IPA. Our numbers are rather encouraging: for markets with 100 schools, this percentage is already very small. With Danish priorities, they are significantly higher.

Many important matching markets are dynamic, so our results provide a guidance on how to organize some of these markets in practice. Some of the most notable examples are the assignment of teachers to public schools, and children to day care centers. Despite the wide range of important applications, the dynamic centralized matching literature is still rather small, specially if compared to the literature on static matching, including the well-known school-choice literature. Most of the results in this dynamic matching literature have been negative, including our own impossibility result (Kennes, Monte, and Tumennasan 2014). Our current paper shows that despite failing strategy-proofness, the DA-IP mechanism might be successfully implemented in large markets.

Here we considered two classes of priority systems: IPA and Danish priorities. It would be interesting to have a maximal domain result concerning the type of priority structures that guarantee strategy-proofness in a dynamic matching market.

\section{Appendix A: Static Stability in Small Economies}

To prove Proposition 1, we need some new definitions and results, which we include in Appendices A and B. The proof of Proposition 1 is in Appendix C.

Fix a finite economy $E=(I, r, X)$ and a period $t-1$ matching $\mu_{t-1}$ of this economy. Now let us construct a new period- $t$ finite economy $E_{t}\left(\mu_{t-1}\right)$ based on our original economy and $\mu_{t-1}$. In this new economy the set of agents is $I_{t} \cup I_{t-1}$ and each agent $i$ is defined by a pair $\left(P_{i}\left(\mu_{t-1}\right), X_{i}\left(\mu_{t-1}\right)\right)$.

Observe that no two agents $i$ and $j$ in this static period- $t$ economy $E_{t}\left(\mu_{t-1}\right)$ can have the same priority score which is less than 1, i.e., it cannot be that $X_{i}^{s}\left(\mu_{t-1}\right)=X_{j}^{s}\left(\mu_{t-1}\right)<1$ for any $s$.

DEFINITION 9 (Static Stablility): We say that school-agent pair $(s, i)$ blocks a period $t$ matching $\mu_{t}$ in economy $E_{t}\left(\mu_{t-1}\right)$ if there exists a school-agent pair $(s, i)$ such that:

(i) $s P_{i}\left(\mu_{t-1}\right) \mu_{t}(i)$,

(ii) $\left|\mu_{t}(s)\right|<r^{s}$ or/and $X_{i}^{s}\left(\mu_{t-1}\right)>X_{j}^{s}\left(\mu_{t-1}\right)$ for some $j \in \mu_{t}(s)$.

A matching $\mu_{t}$ is statically stable in economy $E\left(\mu_{t-1}\right)$ if no school-agent pair blocks $\mu_{t}$.

From Gale and Shapely (1962) each period $t \geq 0$ DA-IP matching $\eta_{t}$ is statically stable in economy $E_{t}\left(\eta_{t-1}\right)$. 
We state the following lemma which is needed later in the online Appendix D.

LEMMA 6: Consider two static economies in period $t, E_{t}$ and $E_{t}^{\prime}$, which are identical except that the set of schools preferred to some school s for some agent $i$ in economy $E_{t}$ is a subset of the one in economy $E_{t}^{\prime}$. Let $\mu_{t}^{\prime}$ be a statically stable matching in $E_{t}^{\prime}$. If $\mu_{t}^{\prime}(i)=s$, then $\mu_{t}^{\prime}$ is a statically stable matching in $E_{t}$.

\section{PROOF:}

Recall that every agent has the same priority score in the two economies. In addition, each agent $j \neq i$ has the same isolated preferences in the two economies. By combining these with the fact that $\mu_{t}^{\prime}$ is stable in economy $E^{\prime}$, we obtain that no school $s^{\prime}$ and agent $j \neq i$ can block $\mu_{t}^{\prime}$ in economy $E_{t}$. Suppose that $i$ and some school $s^{\prime}$ blocks $\mu_{t}^{\prime}$ in economy $E_{t}$. Then $i$ must have a higher priority at $s^{\prime}$ in $E_{t}$. Then, by the conditions given in the lemma, $i$ must have a higher priority at $s^{\prime}$ in $E_{t}^{\prime}$. Therefore, $i$ and $s^{\prime}$ should have been able to block $\mu_{t}^{\prime}$ in $E_{t}^{\prime}$, which contradicts that $\mu_{t}^{\prime}$ is stable in $E_{t}^{\prime}$. Thus, $i$ cannot be a part of a blocking pair.

\section{Appendix B: Static Stability in Continuum Economies}

Let us fix an economy $(\bar{\nu}, \bar{r})$ satisfying Assumption 5. Fix any period $t \geq 0$ and a period $t-1$ matching $\bar{\mu}_{t-1}$. Now let us construct a new period $t$ continuum economy $\bar{F}_{t}\left(\mu_{t-1}\right)=\left(\bar{\nu}_{t}, \bar{r}\right)$ based on our original economy and $\bar{\mu}_{t-1}$. In this new economy the set of agents is $\bar{I}_{t} \cup \bar{I}_{t-1}$ and each agent $i$ is defined by a pair $\left(\bar{P}_{i}\left(\bar{\mu}_{t-1}\right), \bar{X}_{i}\left(\bar{\mu}_{t-1}\right)\right)$. With the new notations, $\bar{I}_{t} \cup \bar{I}_{t-1}$ is distributed on $\overline{\mathcal{P}} \times[0,1]^{n}$ according to a measure $\bar{\nu}_{t}$, where

$$
\begin{aligned}
& \bar{\nu}_{t}\left(\left\{i \in \bar{I}_{t-1} \cup \bar{I}_{t}: x \leq \bar{X}_{i}\left(\bar{\mu}_{t-1}\right) \leq x^{\prime}\right\}\right) \\
& \quad=\bar{\nu}\left(\left\{i \in I_{t-1}: x \leq \bar{X}_{i}\left(\bar{\mu}_{t-1}\right) \leq x^{\prime}\right\}\right)+\bar{\nu}\left(\left\{i \in I_{t}: x \leq \bar{X}_{i}\left(\bar{\mu}_{t-1}\right) \leq x^{\prime}\right\}\right)
\end{aligned}
$$

for all $x, x^{\prime} \in[0,1]^{n}$ where $x \ll x^{\prime}$.

Let $\overline{\mathcal{P}}$ be the all possible rankings of $S$. Now observe that $\bar{\nu}_{t}$ has a full support because by Assumption 5 it must be that

$$
\bar{\nu}\left(\left\{i \in I_{t}: \bar{P}=\bar{P}\left(\mu_{t-1}\right) \text { and } x \leq \bar{X}_{i}\left(\bar{\mu}_{t-1}\right) \leq x^{\prime}\right\}\right)>0
$$

for all $\bar{P} \in \mathcal{P}$ and $x, x^{\prime} \in[0,1]^{n}$ where $x \ll x^{\prime}$. In addition, $\bar{\nu}_{t}\left(\left\{i \in \hat{I}_{t}: X_{i}^{s}\left(\bar{\mu}_{t-1}\right)=x\right\}\right)=0$ for all $x<1$ and $s \in S$.

DEFINITION 10: Period t matching $\bar{\mu}_{t}$ is statically stable in economy $\bar{F}_{t}\left(\bar{\mu}_{t-1}\right)$ if there exists no school-agent pair $(s, i)$ such that:

(i) $s \bar{P}_{i}\left(\bar{\mu}_{t-1}\right) \bar{\mu}_{t}(i)$

(ii) $\hat{\nu}\left(\mu_{t}(s)\right)<\bar{r}^{s}$ or/and $\bar{X}_{i}^{s}\left(\bar{\mu}_{t-1}\right)>\bar{X}_{j}^{s}\left(\bar{\mu}_{t-1}\right)$ for some $j \in \bar{\mu}_{t}(s)$. 
LEMMA 7: For any economy $\bar{F}_{t}\left(\bar{\mu}_{t-1}\right)$, there exists a unique statically stable matching.

\section{PROOF:}

We have already pointed out that $\bar{\nu}_{t}$ has a full support and $\bar{\nu}_{t}\left(\left\{i \in \bar{I}_{t-1} \cup \bar{I}_{t}: \bar{X}_{i}^{s}\left(\bar{\mu}_{t-1}\right)=x\right\}\right)=0$ for all $x<1$ and $s \in S$. Therefore, all the requirements for Theorem 1 of Azevedo and Leshno (2016) are satisfied, hence $\bar{F}_{t}\left(\bar{\mu}_{t-1}\right)$ has a unique statically stable matching.

LEMMA 8: For any economy $\bar{F}_{t}\left(\bar{\eta}_{t-1}\right), \bar{\eta}_{t}$ is a unique statically stable matching.

\section{PROOF:}

This follows from Lemma 8 and Proposition A1 of Azevedo and Leshno (2016)

\section{ApPendix C: Proofs}

\section{PROOF OF LEMMA 1:}

First, let us show that any agent born in period 0 cannot manipulate the DA-IP mechanism. To see this, recall that these agents' matching in period 0 is exogenously determined and to determine the period-1 matchings, the DA-IP mechanism uses the isolated preferences. In addition, because the DA mechanism is strategy proof in static settings, by misreporting no agent born in period 0 improves in terms of her isolated preferences. Thus, we shall prove the three relations below:

(2) $\left(\hat{\eta}_{t_{i}+1}(i), \hat{\eta}_{t_{i}+1}(i)\right) \underbrace{\succ_{i}}_{1}(\left(\eta_{t_{i}+1}(i), \eta_{t_{i}+1}(i)\right) \underbrace{\succeq_{i}}_{2}\left(\eta_{t_{i}}(i), \eta_{t_{i}}(i)\right) \underbrace{\succeq_{i}}_{3}\left(\hat{\eta}_{t_{i}}(i), \hat{\eta}_{t_{i}}(i)\right)$.

To prove Relation 3 in (1), observe that the DA-IP - a strategy-proof mechanism in static settings-yields $\eta_{t_{i}}(i)$ under truth telling and $\hat{\eta}_{t_{i}}(i)$ under the manipulation. Consequently, $\eta_{t_{i}}(i) P_{i}\left(\eta_{t_{i}-1}\right) \hat{\eta}_{t_{i}}(i)$, which is Relation 3 .

Agent $i$ has the highest priority at school $\eta_{t_{i}}(i)$ in period $t_{i}+1$. Thus, the definitions of isolated preferences and DA-IP yield that

$$
\eta(i) \succeq_{i}\left(\eta_{t_{i}}(i), \eta_{t_{i}}(i)\right)
$$

The relation above and Assumption 1 yield Relation 2 in (1).

Now we show Relation 1. On contrary suppose $\left(\eta_{t_{i}+1}(i), \eta_{t_{i}+1}(i)\right)$ $\succeq_{i}\left(\hat{\eta}_{t_{i}+1}(i), \hat{\eta}_{t_{i}+1}(i)\right)$. First observe that $\hat{\eta}_{t_{i}+1}(i) \neq \hat{\eta}_{t_{i}}(i)$. Otherwise, Relation 3 and (3) yield that $\eta(i) \succeq_{i} \hat{\eta}(i)$ which is a contradiction. Because $\hat{\eta}_{t_{i}+1}(i) \neq \hat{\eta}_{t_{i}}(i)$, Assumption 1 and Relation 3 yield that

$$
\left(\eta_{t_{i}}(i), \hat{\eta}_{t_{i}+1}(i)\right) \succeq_{i} \hat{\eta}(i)
$$


Now observe that $\hat{\eta}_{t_{i}+1}(i) \neq \eta_{t_{i}}(i)$. Otherwise, (4) gives that $\left(\eta_{t_{i}}(i), \eta_{t_{i}}(i)\right) \succeq_{i} \hat{\eta}(i)$. This and (3) give that $\eta(i) \succeq_{i} \hat{\eta}(i)$, which is a contradiction. Because $\eta_{t_{i}}(i) \neq \hat{\eta}_{t_{i}+1}(i)$, the supposition and Assumption 1 give

$$
\eta(i) \succeq_{i}\left(\eta_{t_{i}}(i), \hat{\eta}_{t_{i}+1}(i)\right)
$$

This and (4) yield that $\hat{\eta}_{t_{i}+1}(i)=\eta_{t_{i}+1}(i)$. Then the fact that $\hat{\eta}_{t_{i}+1}(i) \neq \hat{\eta}_{t_{i}}(i)$, Relation 3 and Assumption 1 yield that $\eta(i) \succeq \hat{\eta}(i)$ which is a contradiction.

\section{PROOF OF PROPOSITION 1:}

Take any sequence of DA matchings $\left\{\eta^{k}\right\}$ and the corresponding sequence of threshold scores $\left\{\mathbf{p}^{k}\right\}$. For this proof we will use an induction argument. Assume that for all $\tau=0, \ldots, t-1, \mathbf{p}_{\tau}^{k} \rightarrow_{k \rightarrow \infty} \overline{\mathbf{p}}_{\tau}$. At $t=1$, this is definitely true because $\mathbf{p}_{0}^{k}=0$ and $\overline{\mathbf{p}}_{0}=0$. Now we show $\mathbf{p}_{t}^{k} \rightarrow_{k \rightarrow \infty} \overline{\mathbf{p}}_{t}$.

As in Appendices $\mathrm{A}$ and $\mathrm{B}$, we construct period- $t$ economies $\left\{E_{t}^{k}\left(\eta_{t-1}^{k}\right)\right\}$ and $\bar{F}_{t}\left(\bar{\eta}_{t-1}\right)$. Now, based on $E_{t}^{k}\left(\eta_{t-1}^{k}\right)$, let us define economy $\tilde{F}_{t}^{k}\left(\eta_{t-1}^{k}\right)=(\tilde{\nu}, \tilde{r})$, where the measure $\tilde{\nu}$ is a measure satisfying $\tilde{\nu}(\{i\})=1 /\left|I_{0}^{k}\right|$, and $\tilde{r}=r /\left|I_{0}^{k}\right|$. Because $\eta_{t-1}^{k} \rightarrow_{k \rightarrow \infty} \bar{\eta}_{t-1}$, any sequence $\left\{\mathbf{p}_{t-1}^{k}\right\}$ converges to $\overline{\mathbf{p}}_{t-1}$. Consequently, the sequence of measures $\tilde{\nu}_{t}^{k}$ must converge to $\bar{\nu}_{t}$ in the weak ${ }^{*}$ sense. Then Theorems 2(ii) and 2(iii) of Azevedo and Leshno (2016) give that $\mathbf{p}_{t}^{k} \rightarrow \overline{\mathbf{p}}_{t}$. This completes the proof.

\section{PROOF OF THEOREM 4:}

Suppose that agent $i$ in finite economy $E$ can manipulate the DA-IP mechanism. Let agent $i$ 's DA-IP matchings in economy $E$ and at the successful manipulation be $\eta(i)$ and $\hat{\eta}(i)$, respectively. Let $\mathbf{p}$ and $\hat{\mathbf{p}}$ be the threshold scores corresponding to $\eta$ and $\hat{\eta}$, respectively.

Lemma 1 implies that agent $i$ is not matched to $s \equiv \hat{\eta}_{t_{i}+1}(i)$ in period $t_{i}$ at both matchings $\hat{\eta}$ and $\eta$. Thus, $X_{i}^{s}\left(\eta_{t-1}\right)=X_{i}^{s}\left(\hat{\eta}_{t-1}\right)=x_{i}^{s}$. Then because $i$ is matched to $s$ at period $t_{i}+1$ under $\hat{\eta}$ but not under $\eta$, it must be that

$$
\hat{p}_{t_{i}+1}^{s} \leq x_{i}^{s}<p_{t_{i}+1}^{s}
$$

In other words, if an agent $i$ can manipulate the DA-IP mechanism then there must exist a school $s \in S$ such that the inequality above is satisfied. Therefore, to prove the theorem it suffices to show that at each $t \geq 1, s, \epsilon>0$, there exists large enough $\bar{k}$ such that for all $k \geq \bar{k}$, there exists no agent with $t_{i}=t-1$, $\left|x_{i}^{s}-\bar{p}_{t}^{s}\right| \geq \epsilon$ and $\hat{p}_{t}^{s k} \leq x_{i}^{s}<p_{t}^{s k}$.

Suppose that the statement above is false. This means that for some $t \geq 1$, $s, \epsilon>0$ and any $\bar{k}$, there exists $k \geq \bar{k}$ and $i$ with $t_{i}=t-1,\left|x_{i}^{s}-\bar{p}_{t}^{s}\right| \geq \epsilon$ and $\hat{p}_{t}^{s k} \leq x_{i}^{s}<p_{t}^{s k}$. In other words, we can choose a subsequence of economies $E^{k_{j}}$, 
such that in each economy in this sequence, there exists agent $i^{k_{j}}$ who is born in period $t-1,\left|x_{i}^{k_{j}}-\bar{p}_{t}^{s}\right| \geq \epsilon$ and $\hat{p}_{t}^{s k_{j}} \leq x_{i^{k_{j}}}^{s}<p_{t}^{s k_{j}}$. Clearly, $\left\{E^{k_{j}}\right\}$ converges to $\bar{F}$ in weak ${ }^{*}$ sense. This means that $p_{t}^{s k_{j}}$ must converge to $\bar{p}_{t}^{s}$. Now consider the sequence of finite economies $\hat{E}^{k_{j}}$, which differs from $E^{k_{j}}$ only in that the preferences of agent $i^{k_{j}}$ is the same as the the preferences reported at the successful manipulation. Because in each of these economies only one agent's preferences are changed, $\hat{E}^{k_{j}}$ converges to $\bar{F}$ in weak ${ }^{*}$ sense. This means that $\left\{\hat{p}_{t}^{s k_{j}}\right\}$ must converge to $\bar{p}_{t}^{s}$. Recall that we already showed that $p_{t}^{s k_{j}}$ converges to $\bar{p}_{t}^{s}$. This means that as $k_{j}$ increases, $x_{i^{k_{j}}}^{s}$ must be arbitrarily close to $\bar{p}_{t}^{s}$ because $\hat{p}_{t}^{s k_{j}} \leq x_{i^{k_{j}}}^{s}<p_{t}^{s k_{j}}$. Therefore, for a high enough $k_{j}$ it cannot be $\left|x_{i^{k_{j}}}^{s}-\bar{p}_{t}^{s}\right| \geq \epsilon$ which is a contradiction.

This completes the proof as $\bar{\nu}\left(\left\{i: t_{i}=t\right.\right.$ and $\left.\left.x_{i}^{s}=e\right\}\right)=0$ for any $t$ and $e \in[0,1)$.

\section{REFERENCES}

Abdulkadiroğlu, Atila, Yeon-Koo Che, and Yosuke Yasuda. 2015. "Expanding 'Choice' in School Choice." American Economic Journal: Microeconomics 7 (1): 1-42.

Azevedo, Eduardo M., and Eric Budish. Forthcoming. "Strategy-Proofness in the Large." Review of Economic Studies.

Azevedo, Eduardo M., and John W. Hatfield. 2013. "Complementarity and Multidimensional Heterogeneity in Large Matching Markets.” Unpublished. http://econ-server.umd.edu/ davis/eventpapers/ HatfieldHeterogeneity.pdf.

Azevedo, Eduardo M., and Jacob D. Leshno. 2016. "A Supply and Demand Framework for Two-Sided Matching Markets." Journal of Political Economy 124 (5): 1235-68.

-Bloch, Francis, and David Cantala. 2013. "Markovian Assignment Rules." Social Choice and Welfare 40 (1): $1-25$.

Che, Yeon-Koo, and Fuhito Kojima. 2010. "Asymptotic Equivalence of Probabilistic Serial and Random Priority Mechanisms." Econometrica 78 (5): 1625-72.

Che, Yeon-Koo, and Olivier Tercieux. Forthcoming. "Efficiency and Stability in Large Matching Markets." Journal of Political Economy.

Compte, Olivier, and Philippe Jehiel. 2008. "Voluntary Participation and Reassignment in Two-Sided Matching." Unpublished. http://www.enpc.fr/ceras/compte/matchingparticipation.pdf.

Damiano, Ettore, and Ricky Lam. 2005. "Stability in Dynamic Matching Markets." Games and Economic Behavior 52 (1): 34-53.

Doval, Laura. 2018. "A Theory of Stability in Dynamic Matching Markets." Unpublished. http://www. laura-doval.com/a-theory-of-stability-in-dynamic-matching-markets.

Dubins, Lester E., and David A. Freedman. 1981. "Machiavelli and the Gale-Shapley Algorithm." American Mathematical Monthly 88 (7): 485-94.

Dur, Umut. 2011. "Dynamic School Choice." Unpublished. http://www.gtcenter.org/Archive/2011/ Conf/Dur1312.pdf.

-Gale, David, and Lloyd S. Shapley. 1962. "College Admissions and the Stability of Marriage." American Mathematical Monthly 69 (1): 9-15.

Immorlica, Nicole, and Mohammad Mahdian. 2005. "Marriage, Honesty, and Stability." In Proceedings of the Sixteenth Annual ACM-SIAM Symposium on Discrete Algorithms (SODA), 53-62. Vancouver, BC: Association for Computer Machinery, Inc., and Society for Industrial and Applied Mathematics.

-Kadam, Sangram, and Maciej Kotowski. 2018. "Multi-period Matching." International Economic Review 59 (4): 1927-47.

Kennes, John, Daniel Monte, and Norovsambuu Tumennasan. 2014. "The Day Care Assignment: A Dynamic Matching Problem.” American Economic Journal: Microeconomics 6 (4): 362-408.

Kennes, John, Daniel Monte, and Norovsambuu Tumennasan. 2019. "Strategic Performance of Deferred Acceptance in Dynamic Matching Problems: Dataset." American Economic Journal: Microeconomics. https://doi.org/10.1257/mic.20170077. 
Kojima, Fuhito. 2010. "Impossibility of Stable and Non-bossy Matching Mechanisms." Economics Letters 107: 69-70.

-Kojima, Fuhito, and Mihai Manea. 2010. "Incentives in the Probabilistic Serial Mechanism.” Journal of Economic Theory 145 (1): 106-23.

-Kojima, Fuhito, and Parag A. Pathak. 2009. "Incentives and Stability in Large Two-Sided Matching Markets." American Economic Review 99 (3): 608-27.

-Kojima, Fuhito, Parag A. Pathak, and Alvin E. Roth. 2013. "Matching with Couples: Stability and Incentives in Large Markets." Quarterly Journal of Economics 128 (4): 1585-1632.

Kurino, Morimitsu. 2008. "Credibility, Efficiency and Stability: A Theory of Dynamic Matching Markets." Unpublished. http://www.accessecon.com/pubs/SCW2008/GeneralPDFSCW2008/ SCW2008-08-00075S.pdf.

Kurino, Morimitsu. 2009. "Essays on Dynamic Matching Markets." PhD diss. University of Pittsburgh.

-Kurino, Morimitsu. 2014. "House Allocation with Overlapping Generations." American Economic Journal: Microeconomics 6 (1): 258-89.

Lee, SangMok. 2017. "Incentive Compatibility of Large Centralized Matching Markets." Review of Economic Studies 84 (1): 444-63.

Liu, Qingmin, and Marek Pycia. 2016. "Ordinal Efficiency, Fairness, and Incentives in Large Markets." Unpublished. http://pycia.bol.ucla.edu/liu-pycia-large.pdf.

Manea, Mihai. 2009. "Asymptotic Ordinal Inefficiency of Random Serial Dictatorship." Theoretical Economics 4 (2): 165-97.

-McVitie, D.G., and L.B. Wilson. 1971. "Three Procedures for the Stable Marriage Problem." Communications of the ACM 14 (7): 491-92.

-Monte, Daniel, and Norovsambuu Tumennasan. 2015. "Centralized Allocation in Multiple Markets." Journal of Mathematical Economics 61: 74-85.

-Pápai, Szilvia. 2000. "Strategyproof Assignment by Hierarchical Exchange.” Econometrica 68 (6): 1403-33.

-Peranson, Elliott, and Alvin E. Roth. 1999. "The Redesign of the Matching Market for American Physicians: Some Engineering Aspects of Economic Design.” American Economic Review 89 (4): $748-80$.

-Pereyra, Juan Sebastian. 2013. “A Dynamic School Choice Model.” Games and Economic Behavior 80: $100-114$.

Roth, Alvin E., and Marilda A.O. Sotomayor. 1990. Two-Sided Matching: A Study in Game-Theoretic Modeling and Analysis. Cambridge: Cambridge University Press.

—Ünver, M. Utku. 2010. "Dynamic Kidney Exchange." Review of Economic Studies 77 (1): 372-414. 
This article has been cited by:

1. Maciej H. Kotowski. 2019. A Perfectly Robust Approach to Multiperiod Matching Problems. SSRN Electronic Journal . [Crossref]

2. Sangram V. Kadam, Maciej H. Kotowski. 2018. Time horizons, lattice structures, and welfare in multi-period matching markets. Games and Economic Bebavior 112, 1-20. [Crossref]

3. Sangram V. Kadam, Maciej H. Kotowski. 2018. MULTIPERIOD MATCHING. International Economic Review 59:4, 1927-1947. [Crossref] 